\title{
A machine learning based sensitivity analysis of the GTN damage parameters for dynamic fracture propagation in X70 pipeline steel
}

\author{
Benoît Paermentier, Dimitri Debruyne, Reza Talemi* \\ Department of Materials Engineering, KU Leuven, Leuven, Belgium \\ reza.hojjatitalemi@kuleuven.be
}

\begin{abstract}
The Gurson-Tvergaard-Needleman (GTN) damage model is widely used to predict ductile failure initiation and propagation. However, the material-dependent parameters can show a significant spread when determined for the same steel grade material. Different calibration procedures and optimisation processes cause a significant variation in the obtained parameter values. Furthermore, there is no clear consensus on which parameters require calibration. In this study, the influence of the material-dependent parameters used to model the dynamic ductile fracture behaviour of X70 grade pipeline steel is investigated. A sensitivity analysis is performed on a finite element model of a Charpy V-Notch (CVN) specimen. Seven GTN model parameters are considered in a total of 70 simulations. A feedforward back-propagating artificial neural network (ANN) is constructed and trained using data obtained through the numerical simulations. A Connected Weights $(\mathrm{CW})$ algorithm allows to determine the relative influence of each parameter on the fracture energy. It was observed that the void growth acceleration factor plays an important role with respect to the parameter influences. Remarkably, the mean nucleation strain, $\varepsilon_{N}$ has the highest relative importance whilst the critical void volume fraction, $f_{c}$ - which is considered as a crucial damage parameter-showed the smallest influence when the acceleration factor is low. On the contrary, when considering a high acceleration factor, $f_{c}$ becomes the most influential parameter. Based on the obtained importance for each parameter, it is suggested that parameters $f_{0}, f_{c}, f_{F}$, and $f_{N}$ should be selected for calibration in each individual application. Finally, the applied machine learning approach is used to predict the fracture energy for a given set of damage parameters for X70 grade steel. It is observed that the trained neural network is able to provide a satisfactory approximation of the CVN fracture energy.
\end{abstract}

Keywords: CVN; Crack Propagation; Ductile Fracture; FEM; GTN; Machine Learning 


\begin{tabular}{|c|c|}
\hline Nomencla & \\
\hline Acronyms & \\
\hline $\mathrm{AN}$ & Axisymmetric Notched \\
\hline ANN & Artificial Neural Network \\
\hline AI & Artificial Intelligence \\
\hline BTCM & Battelle Two Curve Method \\
\hline CMOD & Crack Mouth Opening Displacement \\
\hline CTOD & Crack Tip Opening Displacement \\
\hline CVN & Charpy V-Notch \\
\hline $\mathrm{CW}$ & Connected Weights \\
\hline DWTT & Drop Weight Tear Test \\
\hline G-T & Gurson-Tvergaard \\
\hline GTN & Gurson-Tvergaard-Needleman \\
\hline MSE & Mean Square Error \\
\hline $\mathrm{PE}$ & Predicted Energy \\
\hline PS & Parameter Set \\
\hline RI & Relative Importance \\
\hline SENT & Single Edge Notch Test \\
\hline $\mathrm{TE}$ & Target Energy \\
\hline Symbols & \\
\hline$f$ & Void volume fraction \\
\hline$f *$ & Effective void volume fraction \\
\hline$\dot{f}$ & Evolution of void volume fraction \\
\hline$f_{0}$ & Initial void volume fraction \\
\hline$f_{c}$ & Critical void volume fraction \\
\hline$f_{F}$ & Void volume fraction at failure \\
\hline$f_{N}$ & Void volume fraction of void nucleating particles \\
\hline$\kappa$ & Void growth acceleration factor \\
\hline$n$ & Strain hardening exponent \\
\hline$q_{1}, q_{2}, q_{3}$ & Constitutive GTN damage parameters \\
\hline$s_{N}$ & Standard deviation nucleation strain \\
\hline$w_{x y}$ & Connection weight between input and hidden neuron \\
\hline$w_{y z}$ & Connection weight between hidden and output neuron \\
\hline$D, p$ & Cowper-Symonds coefficients \\
\hline$E$ & Young's modulus \\
\hline$J$ & Fracture energy \\
\hline$K$ & Strength coefficient \\
\hline$R$ & Ratio of dynamic yield stress to static yield stress \\
\hline$\varepsilon_{N}$ & Mean value of nucleation strain \\
\hline$\varepsilon^{p l}$ & Plastic strain \\
\hline $\bar{\varepsilon}^{p l}$ & Equivalent plastic strain \\
\hline
\end{tabular}




\begin{tabular}{|ll|}
\hline$\dot{\varepsilon}^{p l}$ & Plastic strain rate \\
$\dot{\varepsilon}_{k k}^{p l}$ & Rate of plastic volume change \\
$\varepsilon_{m}^{p l}$ & Equivalent plastic strain \\
$v$ & Poisson ration \\
$\sigma_{e q}$ & Equivalent stress \\
$\sigma_{h y d}$ & Hydrostatic stress \\
$\sigma_{y l d}$ & Yield stress \\
$\bar{\sigma}\left(\bar{\varepsilon}^{p l}\right)$ & Flow curve \\
$\Phi$ & Flow potential \\
\hline
\end{tabular}

\section{Introduction}

One of the major concerns in pipeline design is sufficient material resistance against running fractures. Ductile failure of pipelines can have catastrophic consequences and should be avoided at all cost. Fracture control plans provide recommendations for the minimum required toughness to arrest a long running ductile fracture. Since the 1960's, extensive full-scale experiments were conducted by organisations such as the Battelle Memorial Institute in order to study fracture arrest in line pipes $[1,2]$. Even though these tests closely approximate the conditions of an operational pipeline [3], they are too expensive and time-consuming for industrial applications [4]. Consequently, laboratory fracture tests were developed in order to estimate the crack propagation behaviour. The Charpy V-Notch (CVN) is a standardised impact test [5] which is still being used as an economical and relatively easy-to-perform experiment to determine the fracture toughness of a particular material. It is known that the CVN impact test can lead to non-conservative predictions when applied to high-grade steels. In that case, correction factors such as the modified Leis correction [6] should be implemented for each individual case when determining fracture toughness based on CVN-energy [7]. Based on fullscale burst data and lab-scale experiments, empirical fracture arrest criteria such as the wellestablished Battelle Two Curve Method (BTCM) [8] were introduced.

Initially, brittle fracture was the primary concern during the design of gas transmission pipelines. Due to the high yield to tensile ratio and low strain hardening exponent, these highgrade steels have a limited capacity to distribute plastic deformation away from the defect zone $[9,10]$. This results in a limited strain hardening capacity leading to a reduced failure pressure. Due to the ductile nature of fracture propagation, a minimum fracture toughness is required 
[11]. Therefore, in order to qualify for pipeline applications, a material must pass a CVN-energy absorption criterion [12].

Experimental campaigns are often assisted by numerical simulations in order to facilitate the development and optimisation of new materials and products. Finite element methods have been used extensively to simulate dynamic ductile fracture propagation. Especially continuum damage models have been implemented in the recent years. The GTN damage model, which was developed during the early 1980's, has proven to be an adequate tool for the prediction of ductile damage. With the implementation of the GTN model in ABAQUS software - as porous metal plasticity - the GTN model has grown to be a very popular and widely used damage model. Due to a limited number of damage parameters, the calibration procedure is more feasible in comparison to other damage models such as the Modified Bai-Wierzbicki (MBW) model [13]. However, there is no consensus on the optimal procedure for calibration of the material-dependent parameters. First, the type of calibration experiment often differs from study to study. The experiment type should be selected based on the stress state present in the application of interest in order to cover the range of present stress states. As such, common calibration tests are: the hydraulic bulge test [14], axisymmetric notched tensile tests [15, 16, 17], smooth bar tensile tests [18], and Single Edge Notch Tension (SENT) test [19, 20, 21]. Secondly, it is unclear which parameters should be calibrated and which parameters can be considered as a typical constant value. It is a common practice to calibrate one or more parameters and copy the remaining parameters as provided in literature in order to minimise the calibration effort [22]. Moreover, the selection of calibrated parameters and non-calibrated parameters in the available literature is inconsistent. For instance, the constitutive damage parameters of the GTN model - which will be discussed in section 2.2 - are often considered as typical constants whilst other investigations have indicated a dependence on the hardening behaviour [23]. Different calibration procedures can lead to a significant deviation for each parameter value even when considering the same steel grade [16, 17, 20]. Brinnel et al. have discussed the non-uniqueness of the GTN parameter sets and used a qualitative approach to analyse the parameter influence on the simulation results in order to propose a calibration scheme [24]. However, the influence of these different parameter sets for the same material and the relative influence of parameter variations on the fracture energy have not yet been quantified.

The use of artificial intelligence (AI) in the fracture mechanics domain is gaining interest in recent years. The increasing complexity of constitutive relations in numerical material models 
make it difficult to analyse the impact of the individual components. Data-driven approaches such as AI offer an efficient tool to assess complex non-linear relations. The use of a deep learning methodology as a predictive tool can result in a simplification of complex damage simulations which can be beneficial for industrial end-users. Furthermore, it gives the users the possibility of making existing systems smarter and more applicable [25]. The extensive investigation of Abendroth et al. [26] successfully implemented a neural network to identify the GTN damage variables. Using data from finite element simulations, a neural network was trained to fit global load-displacement curves. It was observed that a network with a relatively simple structure is able to show good performance when solving complex non-linear problems.

In this study, the influence of the damage parameters on the fracture energy will be quantified in the case of X70 grade pipeline steel through a numerical investigation of a CVN impact experiment. A sensitivity analysis of the damage constants is performed to assess their influence on the predicted fracture toughness. A machine learning approach is applied in the form of an artificial neural network as to analyse the obtained simulation data. This study attempts to implement a machine learning based analysis in order to identify the relative parameter influence for the GTN damage model. Furthermore, the potential predictive capability of this approach is tested by analysing the neural network output for existing GTN parameter sets. Consequently, the Charpy fracture energy can be approximated without running a separate FE model.

\section{GTN Damage Model}

\subsection{Background}

The process of ductile damage accumulation can be described using the three physical phenomena of nucleation, growth and coalescence of microscopic voids. Besides the preexisting voids that are present in the initial material matrix, new voids are initiated at material defects such as non-metallic inclusions. Two populations of voids can be considered: large particles that nucleate voids at small strains and small particles that nucleate voids at large strains [27]. Nucleation primarily occurs at second phase particles where cohesion forces are lower and decohesion of the particle-matrix interface creates voids [28]. Plastic deformation of the surrounding matrix material initiates the void growth process. The mechanisms of void nucleation and void growth induce a weakening effect which increases with growing plastic strain. When the voids reach a certain size threshold, they tend to coalesce and form micro 
cracks. These microscopic cracks eventually lead to a macroscopic crack or link up with a nearby crack tip, leading to crack propagation and subsequent material failure [4]. The crack extends due to crack-void interactions which are described using the ligament length between the crack tip and a micro void [27]. Figure 1 gives an overview of the ductile damage accumulation using the discussed processes of void nucleation Figure 1 (a), void growth (b), and void coalescence (c) until final failure (d).

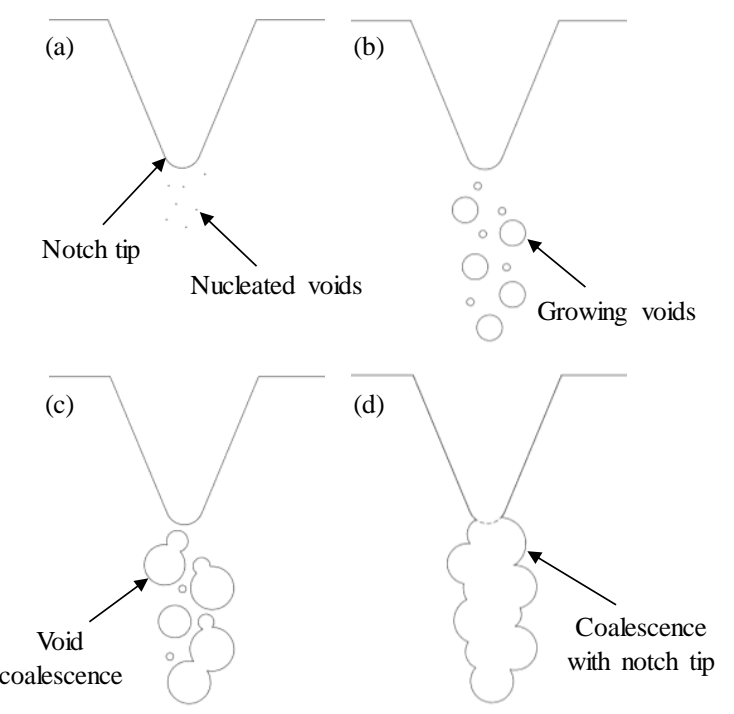

Figure 1: Schematic representation of ductile damage accumulation: presence of initial voids (a), void growth and nucleation of new voids (b); further void growth and void coalescence (c), final coalescence of the voids with nearby crack (d).

A widely accepted and used model to describe this ductile damage process is the GTN model. The fundaments of the GTN model were created by Gurson [29] which considered voids as spherical cavities. Based on the Von Mises yield stress, $\sigma_{y l d}$, Gurson proposed a rateindependent yield condition as a function of the void volume fraction $f$. The resulting plastic flow potential of the material is presented as a function of the macroscopic stress and the void volume fraction.

$$
\Phi=\left(\frac{\sigma_{e q}}{\sigma_{y l d}}\right)^{2}+2 f \cosh \left(\frac{3}{2} \frac{\sigma_{h y d}}{\sigma_{y l d}}\right)-1-f^{2}=0
$$

The Gurson model was modified by Tvergaard [30, 31, 32], introducing constitutive material parameters $q_{1}, q_{2}$, and $q_{3}$ (with $q_{3}=q_{1}^{2}$ ). The adjusted plastic flow potential compensates the overestimation of failure strain in the Gurson model for low stress triaxiality conditions. 


$$
\Phi=\left(\frac{\sigma_{e q}}{\sigma_{y l d}}\right)^{2}+2 q_{1} f \cosh \left(\frac{3}{2} q_{2} \frac{\sigma_{h y d}}{\sigma_{y l d}}\right)-1-q_{3} f^{2}=0
$$

These fitting parameters define previously neglected void interactions. The Gurson-Tvergaard (G-T) model considers the decrease of the load carrying capacity of neighbouring voids as the stress distribution changes. However, due to the introduction of these fitting parameters, the micromechanical background of the G-T model becomes questionable [33].

Even though the Gurson model was adjusted to include void interactions, it still lacked a definition for void coalescence. Tvergaard and Needleman [32] implemented two new material parameters: critical void volume fraction $f_{c}$, and void volume fraction at failure $f_{F}$. The threshold porosity $f_{c}$ defines the onset of void coalescence and $f_{F}$ indicates the void fraction at final failure. This correction approximates the rapid loss of a materials' load carrying capacity to an acceptable extent. The proposed rate-dependent yield function is similar to the G-T model but the void volume fraction was given a different definition.

$$
\Phi=\left(\frac{\sigma_{e q}}{\sigma_{y l d}}\right)^{2}+2 q_{1} f * \cosh \left(\frac{3}{2} q_{2} \frac{\sigma_{b y d}}{\sigma_{y l d}}\right)-1-q_{3} f^{* 2}=0
$$

With

$$
f^{*}=\left\{\begin{array}{ccc}
f & \text { if } & f \leq f_{c} \\
f_{c}+\frac{\bar{f}_{F}-f_{c}}{\left(f_{F}-f_{c}\right)}\left(f-f_{c}\right) & \text { if } & f_{c}<f<f_{F} \\
\bar{f}_{F} & \text { if } & f \geq f_{F}
\end{array}\right.
$$

where the effective void volume fraction $f^{*}$ is a function of void volume fraction $f$ as defined in the G-T model. $\bar{f}_{F}$ is the maximal attainable value for parameter $f$ or $\bar{f}_{F}=1 / q_{1}$. The evolution of void volume fraction is a combination of growth of existing voids and straincontrolled nucleation of new voids [27, 34, 35].

$$
\dot{f}=\dot{f}_{\text {growth }}+\dot{f}_{\text {nucleation }}
$$

Or 
$\dot{f}=(1-f) \dot{\varepsilon}_{k k}^{p l}+\frac{f_{N}}{s_{N} \sqrt{2 \pi}} \exp \left[-\frac{1}{2}\left(\frac{\varepsilon_{m}^{p l}-\varepsilon_{N}}{s_{N}}\right)^{2}\right] \dot{\varepsilon}_{m}^{p l}$

where $\dot{\varepsilon}_{k k}^{p l}$ is the rate of plastic volume change, $\varepsilon_{m}^{p l}$ is the equivalent plastic strain, $f_{N}$ is the volume fraction of void nucleating particles, $s_{N}$ is the standard deviation of the nucleation strain, $f_{N}$ is the volume fraction of nucleated voids, and $\varepsilon_{N}$ is the mean value of the nucleation strain. Consequently, the GTN model has a total of 8 material parameters $\left(q_{1}, q_{2}, f_{0}, f_{c}, f_{F}\right.$, $f_{N}, \varepsilon_{N}$ and $s_{N}$ ) that can describe the ductile damage behaviour of a material. The physical meaning and determination of these parameters is elaborated in following paragraph.

\subsection{Material constants and calibration}

Parameters $q_{1}$ and $q_{2}$ are constitutive values related to the hardening process of the matrix material. The typical values were determined by Tvergaard [36] and many researchers have applied these values as typical constant values when implementing the GTN damage model. However, several studies [36, 23, 37, 38] have reported on numerous dependencies of these qvalues on factors such as material hardening, void shape, plastic strain, etc. Nevertheless, the original values $\left(q_{1}=1.5, q_{2}=1.0\right.$ and $\left.q_{3}=q_{1}^{2}\right)$ determined by Tvergaard are generally used in the literature. However, these values were obtained considering perfect plastic behaviour. Therefore, this ideal approximation neglects hardening as well as time-dependent effects on the plastic material behaviour.

The initial void volume fraction, $f_{0}$ for steels, can be approximated through Franklin's formula which is related to the microstructure of the material [39]. In this case, the total void volume fraction of manganese and sulphide inclusions in the material matrix defines the relative density.

$$
f_{0} \approx 0.054\left(\% S-\frac{0.001}{\% M n}\right)
$$

However, this equation does not consider other non-metallic inclusions (> $1 \mu \mathrm{m}$ ) besides manganese and sulphide oxides that can be attributed to the effective initial void volume fraction. Alternatively, the investigation of Xia et al. [40] indicated that $f_{0}$ does not necessarily represent an observable physical parameter and should be fitted to experimental data. 
The critical void volume fraction, $f_{c}$, is considered as a crucial parameter since it indicates the onset of void coalescence. In other words, $f_{c}$ determines the point at which void volume growth is accelerated by factor $\kappa$.

$$
\kappa=\frac{\left(1 / q_{1}-f_{C}\right)}{f_{F}-f_{C}}
$$

Parameter $f_{c}$ is often obtained through numerical calibration or is determined from unit cell calculations [41]. The void volume fraction at failure, $f_{F}$, indicates the fraction of voids at final failure. Parameter $f_{F}$ can be calibrated by combining experimental and numerical data. Another approach determines solely $f_{F}$ through a microstructure analysis by quantifying the present void fractions at final material failure [42, 43].

Parameters $f_{N}, \varepsilon_{N}$, and $s_{N}$ related to void nucleation and yield locus, were first determined by Chu and Needleman [34], and Tvergaard [36]. The void volume fraction of nucleated voids, $f_{N}$, gives the fraction of newly nucleating voids, not considering the voids that are present in the initial state. Similar to the constitutive parameters, the values for the mean value and standard deviation of the nucleation strain are often considered as typical constants $\left(\varepsilon_{N}=0.3\right.$, $\left.s_{N}=0.1\right)$.

In the literature, a wide variety of calibration experiments and optimisation procedures can be found for the material-dependent parameters. Generally, a series of experiments is conducted and numerical models are created to fit the parameters as good as possible to the experimental data $[44,45,46,47,48,49]$. Other - less conventional - methods implement complex subroutines or artificial neural networks $[26,50]$. The damage parameter sets that are used during this study, were adopted from studies using traditional inverse material characterisation procedures that combine experimental results with numerical fitting.

Most common experiments applied as calibration method for the GTN model are the Axisymmetric Notched (AN) tensile bar, the Single Edge Notch Tension (SENT) test, and Compact Tension (CT) test. Based on the obtained experimental data, a stress-strain or loaddisplacement curve is constructed. Numerical models of the calibration experiments are created and the material-dependent parameters are iteratively adjusted until a good agreement is found between the numerical and experimental data. This process is often automated and implemented 
as a subroutine or updating finite element code as to speed up the optimisation process. Figure 2 shows a calibration procedure using numerical simulations to adjust a certain parameter to fit with experimental data. It should be noted that the shown graph is randomly generated and solely serves the purpose of explaining the fitting procedure.

However, there is no consistency in the selection of parameters that should be calibrated and what optimisation procedure that should applied. In most cases, $f_{C}$ is optimised whilst other parameters are copied from other published studies in the literature. Parameters such as the initial void volume fraction, $f_{0}$, the void volume fraction at failure, $f_{F}$, and the void volume fraction of newly nucleated voids, $f_{N}$, are calibrated using different approaches as mentioned in the introduction. In some cases, $f_{0}$ is calculated using Franklin's formula, in other cases it is obtained through unit cell calculation or it is calibrated using experimental data. Parameter $f_{F}$ can be determined through a microstructure analysis or through an optimisation process using experimental and numerical data. Parameter $f_{N}$ can be assumed to be equal to the value determined in the initial studies conducted by Needleman and Tvergaard [32], it can be calibrated, or it can be estimated from the volume fraction of small particles $(<1 \mu \mathrm{m})$ such as precipitated iron carbides $\left(\mathrm{Fe}_{3} \mathrm{C}\right)$.

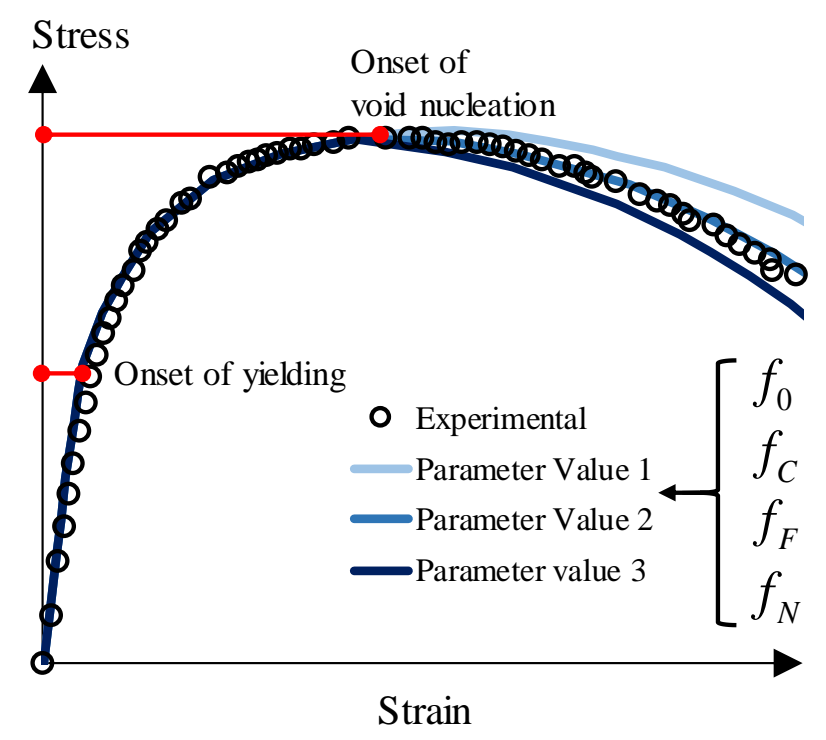

Figure 2: A parameter is adjusted to fit experimental data as a commonly used calibration procedure for the material-dependent GTN parameters, randomised data was used to clarify the example.

Multiple published studies have reported on the calibration of X70 grade steel for the GTN damage model. In each investigation, a set of damage parameters was identified. Four parameter sets (PS) were selected from the literature and are used as reference in this study. PS1 [16] and 
PS3 [17] were obtained through AN tensile bars experiments while PS2 [19] and PS4 [20, 21] were obtained through SENT experiments. It is worth noting that even though in some cases the same calibration experiment was conducted, for each study a parameter set was determined. Variations could be caused by differences in the parameter optimisation procedure. Automated procedures implement algorithms to iteratively determine the GTN constant values. A different selection of algorithm and/or their internal parameters such as stopping tolerance and objective function can result in different calibration results. For example, the study by Rahimidehgolan et al. [44] showed that for different objective functions, different parameter values were obtained. However, extensive comparative studies on the influence of different optimisation algorithms and their respective parameters on the identified values are scarce. At this point it is still unclear if these varying parameter sets also result in a significant deviation of the energy prediction. Furthermore, in every study, different parameters were selected for calibration.

Summarising, the GTN parameters for a material can be determined in different ways: calibrating using a combined numerical-experimental approach, assuming a classic value as determined in the literature, calculating using a formula, or assume a variable relationship. Table 1 gives an overview on how each damage parameter was obtained in the reference sets that are considered during this investigation. It should be noted that for the nucleation parameters for PS3, the limit case of $f_{N}=0$ was selected. Consequently, in case of PS3, parameters $\varepsilon_{N}$ and $s_{N}$ are not considered as they don't yield any physical meaning.

Table 1: Overview of determination methodology for all GTN parameters for X70 grade steel. C: Calibrated, CV: Classic Value, F: Formula, and V: Variable.

\begin{tabular}{c|c|c|cccccccc} 
& Test & $\begin{array}{c}\text { Mesh } \\
{[\mathbf{m m}]}\end{array}$ & $\boldsymbol{q}_{\mathbf{1}}$ & $\boldsymbol{q}_{\mathbf{2}}$ & $\boldsymbol{f}_{\mathbf{0}}$ & $\boldsymbol{f}_{\boldsymbol{C}}$ & $\boldsymbol{f}_{\boldsymbol{F}}$ & $\boldsymbol{f}_{\boldsymbol{N}}$ & $\boldsymbol{\varepsilon}_{\boldsymbol{N}}$ & $\boldsymbol{s}_{\boldsymbol{N}}$ \\
\hline PS1 & $\mathrm{AN}$ & 0.15 & $\mathrm{CV}$ & $\mathrm{CV}$ & $\mathrm{C}$ & $\mathrm{C}$ & $\mathrm{C}$ & $\mathrm{C}$ & $\mathrm{C}$ & $\mathrm{CV}$ \\
PS2 & $\mathrm{SENT}$ & 0.15 & $\mathrm{CV}$ & $\mathrm{CV}$ & $\mathrm{F}$ & $\mathrm{C}$ & $\mathrm{C}$ & $\mathrm{C}$ & $\mathrm{CV}$ & $\mathrm{CV}$ \\
PS3 & $\mathrm{AN}$ & 1 & $\mathrm{CV}$ & $\mathrm{CV}$ & $\mathrm{F}$ & $\mathrm{C}$ & $\mathrm{C}$ & - & - & - \\
PS4 & $\mathrm{SENT}$ & 0.25 & $\mathrm{~F}$ & $\mathrm{~F}$ & $\mathrm{C}$ & $\mathrm{V}$ & $\mathrm{F}$ & $\mathrm{F}$ & $\mathrm{CV}$ & $\mathrm{CV}$
\end{tabular}

The values of the GTN damage parameter in each parameter set are presented in Table 2. To visualise the value range, the minimum and maximum values for each parameter are plotted in Figure 3. 
Table 2: An overview of the absolute values of the determined GTN parameters used for X70 grade steel taken from the literature which are used as the reference sets in this study.

\begin{tabular}{c|cccccccc} 
& $\boldsymbol{q}_{\mathbf{1}}$ & $\boldsymbol{q}_{\mathbf{2}}$ & $\boldsymbol{f}_{\mathbf{0}}$ & $\boldsymbol{f}_{\boldsymbol{C}}$ & $\boldsymbol{f}_{\boldsymbol{F}}$ & $\boldsymbol{f}_{\boldsymbol{N}}$ & $\boldsymbol{\varepsilon}_{\boldsymbol{N}}$ & $\boldsymbol{s}_{\boldsymbol{N}}$ \\
\hline PS1 & 1.5 & 1 & 0.000401 & 0.001517 & 0.5 & 0.067143 & 0.8 & 0.1 \\
PS2 & 1.5 & 1 & 0.000125 & 0.015 & 0.32 & 0.0004 & 0.3 & 0.1 \\
PS3 & 1.5 & 1 & 0.00015 & 0.00074 & 0.18 & 0.0 & - & - \\
PS4 & 1.43 & 0.95 & 0.002 & $0.025-$ & 0.19 & 0.00018 & 0.3 & 0.1
\end{tabular}

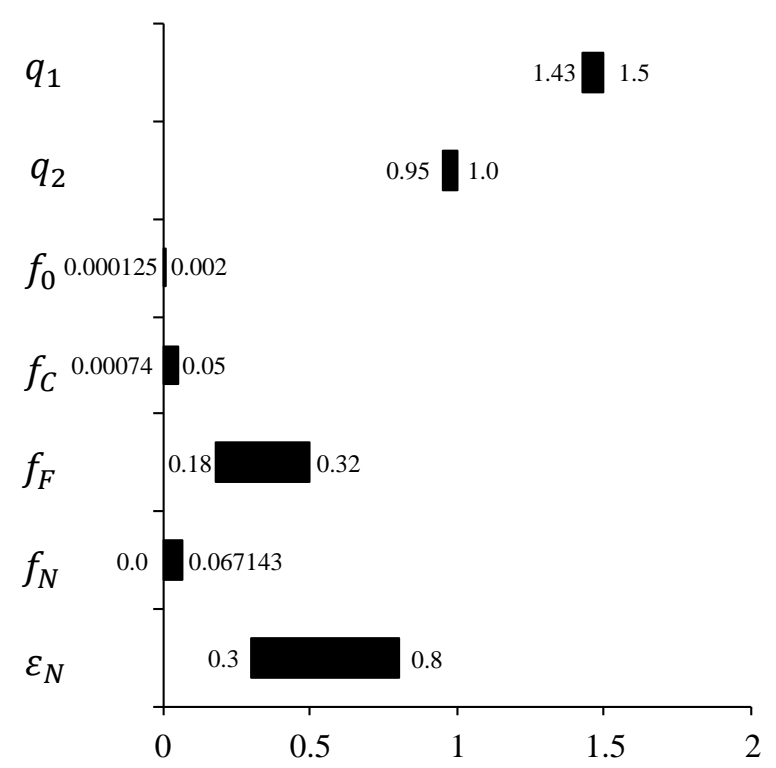

Figure 3: Visualisation of the large value range for the GTN parameters such as $f_{F}$ and $\varepsilon_{N}$ which can be found in the literature for X70 grade pipeline steel.

It can be observed that parameters $f_{0}, f_{C}, f_{F}, f_{N}$, and $\varepsilon_{N}$ show a significant relative spread with up to $50 \%$ of possible variation for a material-dependent parameter when considering the same material. Thus, for X70 grade pipeline steel material, there is a considerable spread of GTN damage parameter values available in the literature. It should be investigated if this also results in a deviation of fracture toughness prediction. Therefore, the relative influence of each damage variable should be investigated. Once the parameters with a significant influence are identified, a statement on the application of the GTN damage model for X70 grade steel can be substantiated. In the next section, a sensitivity analysis is performed in order to assess the importance of each damage parameter - within the available range reported in the literature for $\mathrm{X} 70$ grade steel- with respect to the resulting fracture toughness. As parameter $s_{N}$ does not show any variation, it is not included in the sensitivity analysis. 


\section{Artificial Neural Network (ANN)}

The development of Artificial Intelligence (AI) was initiated by Turing in 1950 [51] and has been a subject of extensive research ever since. Although the concept of intelligence and consciousness of AI are still up for debate [52], the current AI tools have become a practical and efficient tool for data analysis. More specifically, machine learning, has provided data science with the possibility of processing complex and non-linear data. Deep learning is a subset of machine learning which uses a biologically-inspired neural network containing multiple layers of algorithms in order to interpret data relations. An ANN consists out of multiple layers each containing a set of neurons. The concept of a neuron structure is based on the perceptron model of Rosenblatt [53] performing a binary classification of data. However, a neuron uses mathematical functions such as the sigmoid function [54] to grade data inside the binary range of 0 and 1 . Several investigations have successfully implemented machine learning techniques in the field of fracture and damage mechanics using a dataset obtained from FE simulations [26, 50]. These studies have shown that AI-based methods can present satisfactory predictions as well as aid and complement iterative FE modelling campaigns.

In this study, a multilayer perceptron neural network is constructed as a method to determine the relative influences of every material-dependent parameter on the Charpy energy, $J$, as a measure of fracture toughness. Matlab software [55] is used to develop a neural network consisting out of three layers: an input layer containing seven neurons, a hidden layer containing eight neurons, and an output layer containing one neuron. The seven considered parameters are used as input variables whilst the corresponding energy values - obtained through the numerical simulations - are considered as the output of the network. The optimal number of neurons that should be used in the hidden layer is still debatable [56, 57]. Currently, multiple rules of thumb have been proposed but no general consensus has been reached. For networks consisting out of one hidden layer, in most cases an arbitrary number of hidden neurons is selected. In this study, ten hidden neurons were used as it is the default proposed amount by Matlab and no performance anomalies were encountered. A schematic overview of the complete network structure is presented in Figure 4. 


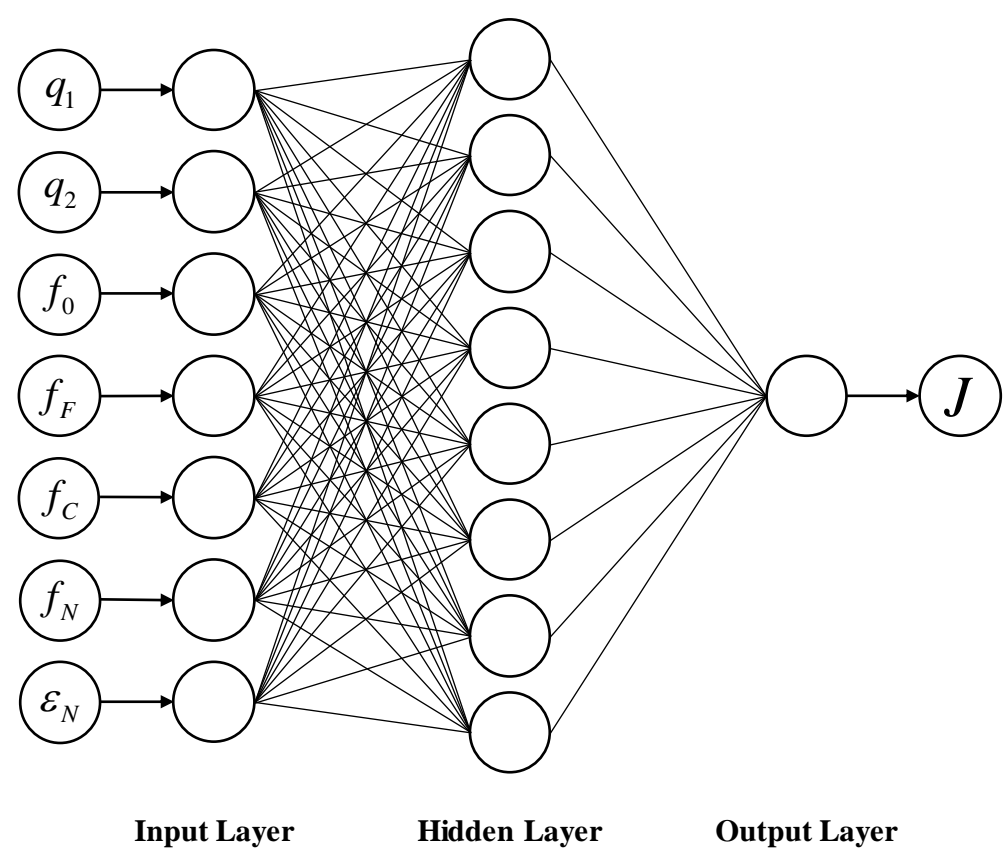

Figure 4: Visualisation of the constructed the multi-layered artificial neural network with seven neurons in the input layer, eight neurons in the hidden layer, and one neuron in the output layer.

Each neuron in a layer is connected to every neuron in previous layers. In other words, the input of every neuron in a layer is the sum of every output of every neuron in previous layers. A weight is assigned to all connections between neurons. The relative weighted sum is obtained by applying a sigmoid function which keeps the activation values of the neuron in the range between 0 and 1 . If the activation of a neuron reaches a certain threshold - referred to as the bias value - the neuron has a meaningful value and is activated. Initially, all weights and biases are randomly generated. By minimising a defined cost function, these weights and biases are optimised. In this study, a mean square error cost function (mse) is defined. Using a backpropagation methodology, based on the gradient descent approach, the cost function is minimised and an optimised neural network is obtained.

$m s e=\frac{1}{N} \sum_{i=1}^{N}\left(e_{i}\right)^{2}$

The relative influence of each variable is determined by assessing the calibrated connection weights using the Connection Weights (CW) methodology [58]. Other frequently used methods such as the Garson algorithm have shown to be less performant and are therefore not considered during this investigation [59]. The Relative Importance ( $R I)$ of each parameter can be analysed based on the obtained connection weights in the neural network. The CW algorithm uses the sum of product for all weights between the input neurons and hidden neurons, and between the hidden layer and output neuron. 


$$
R I_{x}=\sum_{y=1}^{m} w_{x y} w_{y z}
$$

where $R I$ indicates the relative importance of input variable $\mathrm{x}$ as a sum of product of the respective weights $(\mathrm{w})$ between the input layer neurons $(\mathrm{x})$ hidden layer neurons $(\mathrm{y})$, and hidden layer neurons with output neurons (z).

The data obtained from the series of conducted simulations were used to train the network based on the Levenberg-Marquardt algorithm [60,61] which is used to solve the non-linear least squares cost function. All datasets are shuffled randomly as to prevent the training process to be biased. In this study, $70 \%$ of the dataset was used for training whilst the validation and testing procedure each used $15 \%$ of the dataset. In contrast to the training procedure, the validation procedure does not change the connection weights but measures the quality of the generalisation of the network and to halt training when generalisation stop improving. Similar to validation procedure, the testing procedure does not change internal weights but provides an independent measure of the performance during and after training. Based on these results, the neural network can be retrained or can be restructured by adjusting the number of neurons in the hidden layer. In this case, the number of hidden neurons is determined by performing multiple training procedures for ANN's with different number of hidden neurons. The optimal number of hidden neurons is selected based on the lowest remaining error. Consequently, the calibrated network can indicate the sensitivity of the output variable to changes of each input variable. This sensitivity is used to analyse the importance of the material-dependent parameters of the GTN damage model for X70 grade steel. Finally, the trained ANN should be able to predict a fracture energy value for a given GTN parameter set. Therefore, the considered reference sets are used to validate the prediction performance of the network. It is important to note that the reference sets were eliminated from the training data in order to avoid a biased prediction.

\section{Material Properties}

During this study, X70 grade pipeline steel is used to investigate the damage parameter influence. The considered mechanical properties are obtained from the literature [16, 17]. An isotropic hardening relation is used to describe the plasticity behaviour in the post-necking region. Plastic behaviour is considered constant throughout this investigation in order to assess the sensitivity of each material-dependent parameter in the GTN model without the influence of the hardening behaviour. Dynamic material response is considered using the Cowper- 
Symonds overstress power law. This relation takes the strain rate dependency into account by defining the equivalent plastic strain rate $\dot{\bar{\varepsilon}}^{p l}$ as:

$$
\dot{\bar{\varepsilon}}^{\not l}=D(R-1)^{p}
$$

In this equation, $D$ and $P$ are referred to as the Cowper-Symonds coefficients. Neither anisotropy nor adiabatic heating effects were considered throughout this investigation. Table 3 gives an overview of the mechanical properties for X70 grade steel that were implemented for the numerical investigation.

Table 3: Mechanical properties for X70 grade steels as found in the literature $[16,17]$.

\begin{tabular}{ccc}
\hline & & X70 \\
\hline Young's modulus & $E$ & $210 \mathrm{GPa}$ \\
\hline Poisson's ratio & $v$ & 0.3 \\
\hline \multirow{3}{*}{ Isotropic hardening } & $\bar{\sigma}\left(\bar{\varepsilon}^{p l}\right)=\sigma_{y l d}+K\left(\bar{\varepsilon}^{p l}\right)^{n}$ & $\begin{array}{c}\sigma_{y l d}=485 \mathrm{MPa} \\
K=795 \mathrm{MPa} \\
\end{array}$ \\
& & $n=0.13$ \\
\hline \multirow{2}{*}{ Strain rate effect } & $D$ & 55 \\
& $p$ & 5 \\
\hline
\end{tabular}

The plastic material behaviour of X70 grade steel that has been used to obtain parameter sets, i.e. PS1, PS2, PS3, and PS4, is largely the same, as can be seen in Figure 5. Therefore, the plastic input data is not considered as a variable during this investigation. Furthermore, this study focusses on the identification of the most influential GTN damage parameters with respect to the predicted fracture energy. The effect of different plastic behaviour is reduced by keeping the plastic material input constant throughout the investigation. 


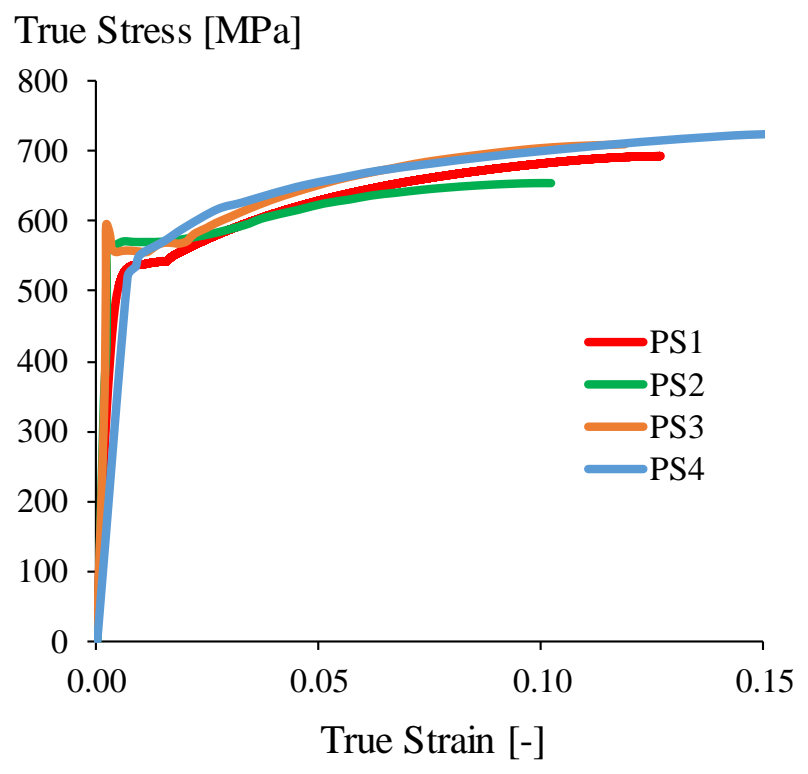

Figure 5: True stress-strain curves used for PS1, PS2, PS3, and PS4 parameter sets for X70 grade steel taken from the literature.

\section{Numerical Model}

\subsection{Loading and Boundary Conditions}

The GTN damage model is implemented in a 3D finite element model using ABAQUS software [62] and linear C3D8R elements with reduced integration. A CVN specimen model is constructed and the damage parameters are introduced accordingly. Due to the symmetry of the CVN test, only one half of the specimen is modelled which results in a reduction of the computational effort. The anvil and striker are modelled as analytical rigid bodies since their stiffness is assumed to be significantly higher than the stiffness of the specimen. An initial velocity of $5.5 \mathrm{~m} / \mathrm{s}$ and a mass of $19.8 \mathrm{~kg}$ are assigned to the reference point of the striker [63]. The loading conditions, boundary conditions and meshing strategy are visualised in Figure 6. 


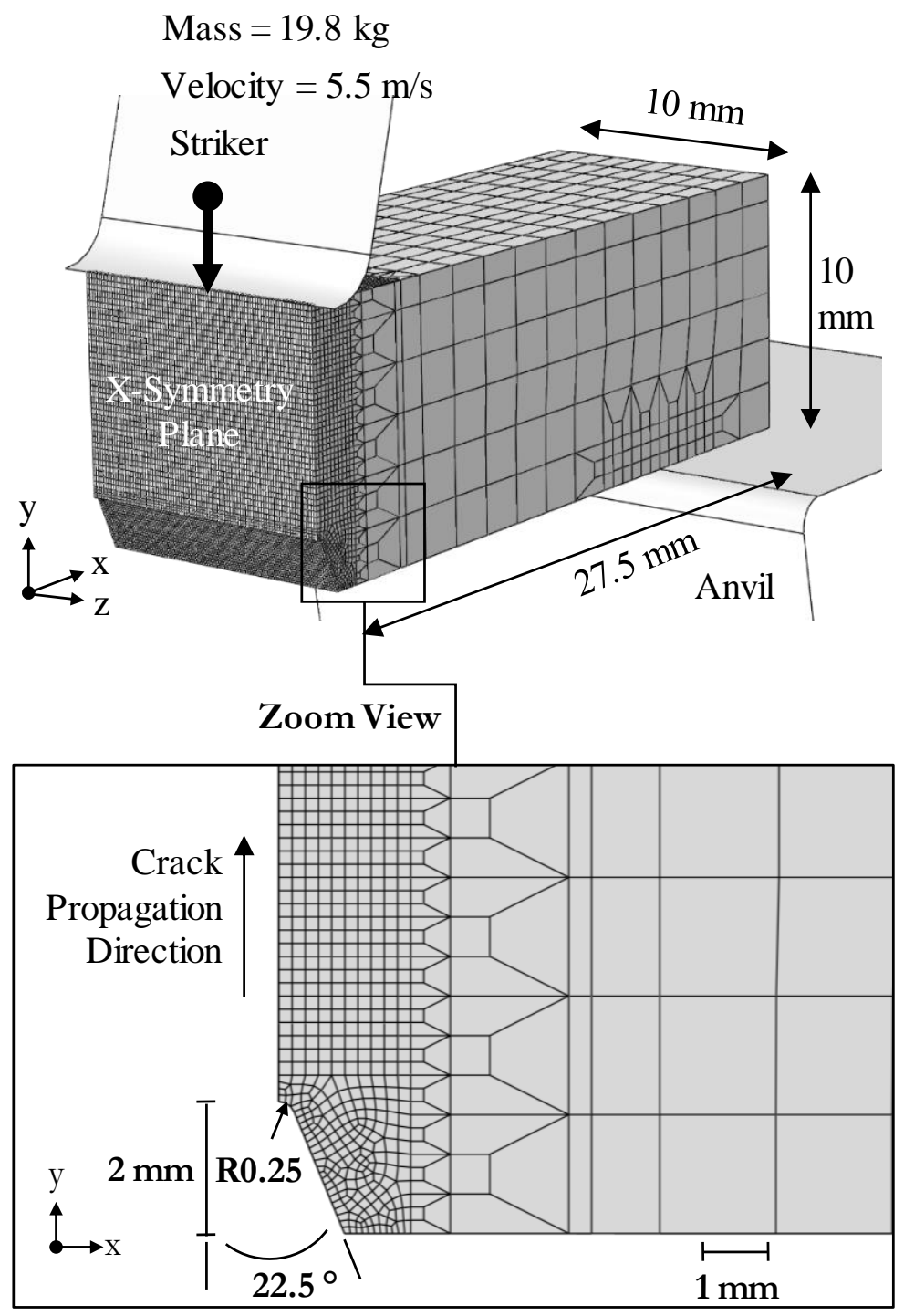

Figure 6: Visualisation of the constructed 3D finite element model of a CVN specimen with corresponding dimensions, boundary conditions, and meshing strategy.

\subsection{Meshing Strategy}

It is well known that the mesh size plays an important role when implementing the GTN model. If a material law includes softening behaviour, the strain field becomes discontinuous near the softening condition and strain tends to concentrate in a small zone - called strain localisation [64]. As strain localisation occurs, the heft of the energy released is proportional to the cell dimension orientated perpendicular to the crack propagation. The element size perpendicular to the crack propagation direction, as indicated in Figure 6, should therefore be selected based on the local fracture process of a particular material. Consequently, the element size is a characteristic length that should be regarded as a material property. 
It has been observed that the determination of this characteristic length is somewhat arbitrary as the cell height has the double role of the RVE (Representative Volume Element) and dimension of the strain localisation zone. In practice, once an element size is chosen, the nucleation parameters and coalescence parameters need to be tuned.

In this work, the characteristic element length for X70 ranges between $0.15 \mathrm{~mm}$ and $0.25 \mathrm{~mm}$ for the gross of the considered reference sets $[16,17,19,20,21]$. The influence of the mesh size over this range has been investigated which resulted in a maximum of $3.5 \%$ deviation in fracture energy. Subsequently, an element size of $0.2 \mathrm{~mm}$ in the fracture region is applied as it showed the most consistent results. As the influence on fracture energy within the considered element size range is rather limited, the element size is fixed to $0.2 \mathrm{~mm}$ and is not considered as a GTN parameter. A structured partitioning strategy was implemented to obtain uniform elements in the fracture region and no changes to the meshing strategy were made throughout the investigation.

\subsection{Sensitivity Analysis Using ANN}

In order to assess which parameters, play a major role in the fracture energy prediction as well as fracture surfaces, a series of simulations are conducted. As PS1 shows best agreement with experimental data [65], this set is selected as the reference set throughout the complete analysis. Every parameter is varied within its value range according to the four reference sets taken from the literature, i.e. PS1 to PS4 as tabulated in Table 2, whilst the remaining parameters are kept constant. This way, the complete realistic range for all considered parameters is covered and the influence of each parameter can be assessed. An overview of all conducted simulations and their respective parameter sets is given in Table 4. 
Table 4: An overview of the performed simulations and their respective parameter values.

\begin{tabular}{|c|c|c|c|c|c|c|c|}
\hline $\begin{array}{c}\text { Simulation } \\
\text { number }\end{array}$ & $q_{1}$ & $q_{2}$ & $f_{0}$ & $\boldsymbol{f}_{C}$ & $\boldsymbol{f}_{F}$ & $f_{N}$ & $\varepsilon_{N}$ \\
\hline Reference & 1.5 & 1.0 & 0.000401 & 0.001517 & 0.5 & 0.067143 & 0.8 \\
\hline 1 & 1.43 & 1.0 & 0.000401 & 0.001517 & 0.5 & 0.067143 & 0.8 \\
\hline 2 & 1.444 & 1.0 & 0.000401 & 0.001517 & 0.5 & 0.067143 & 0.8 \\
\hline 3 & 1.458 & 1.0 & 0.000401 & 0.001517 & 0.5 & 0.067143 & 0.8 \\
\hline 4 & 1.472 & 1.0 & 0.000401 & 0.001517 & 0.5 & 0.067143 & 0.8 \\
\hline 5 & 1.486 & 1.0 & 0.000401 & 0.001517 & 0.5 & 0.067143 & 0.8 \\
\hline 6 & 1.5 & 0.95 & 0.000401 & 0.001517 & 0.5 & 0.067143 & 0.8 \\
\hline 7 & 1.5 & 0.96 & 0.000401 & 0.001517 & 0.5 & 0.067143 & 0.8 \\
\hline 8 & 1.5 & 0.97 & 0.000401 & 0.001517 & 0.5 & 0.067143 & 0.8 \\
\hline 9 & 1.5 & 0.98 & 0.000401 & 0.001517 & 0.5 & 0.067143 & 0.8 \\
\hline 10 & 1.5 & 0.99 & 0.000401 & 0.001517 & 0.5 & 0.067143 & 0.8 \\
\hline 11 & 1.5 & 1.0 & 0.00125 & 0.001517 & 0.5 & 0.067143 & 0.8 \\
\hline 12 & 1.5 & 1.0 & 0.000875 & 0.001517 & 0.5 & 0.067143 & 0.8 \\
\hline 13 & 1.5 & 1.0 & 0.0005 & 0.001517 & 0.5 & 0.067143 & 0.8 \\
\hline 14 & 1.5 & 1.0 & 0.000125 & 0.001517 & 0.5 & 0.067143 & 0.8 \\
\hline 15 & 1.5 & 1.0 & 0.000401 & 0.00074 & 0.5 & 0.067143 & 0.8 \\
\hline 16 & 1.5 & 1.0 & 0.000401 & 0.010592 & 0.5 & 0.067143 & 0.8 \\
\hline 17 & 1.5 & 1.0 & 0.000401 & 0.020444 & 0.5 & 0.067143 & 0.8 \\
\hline 18 & 1.5 & 1.0 & 0.000401 & 0.030296 & 0.5 & 0.067143 & 0.8 \\
\hline 19 & 1.5 & 1.0 & 0.000401 & 0.040148 & 0.5 & 0.067143 & 0.8 \\
\hline 20 & 1.5 & 1.0 & 0.000401 & 0.05 & 0.5 & 0.067143 & 0.8 \\
\hline 21 & 1.5 & 1.0 & 0.000401 & 0.001517 & 0.18 & 0.067143 & 0.8 \\
\hline 22 & 1.5 & 1.0 & 0.000401 & 0.001517 & 0.244 & 0.067143 & 0.8 \\
\hline 23 & 1.5 & 1.0 & 0.000401 & 0.001517 & 0.308 & 0.067143 & 0.8 \\
\hline 24 & 1.5 & 1.0 & 0.000401 & 0.001517 & 0.372 & 0.067143 & 0.8 \\
\hline 25 & 1.5 & 1.0 & 0.000401 & 0.001517 & 0.436 & 0.067143 & 0.8 \\
\hline 26 & 1.5 & 1.0 & 0.000401 & 0.001517 & 0.5 & 0 & 0.8 \\
\hline 27 & 1.5 & 1.0 & 0.000401 & 0.001517 & 0.5 & 0.013429 & 0.8 \\
\hline 28 & 1.5 & 1.0 & 0.000401 & 0.001517 & 0.5 & 0.026857 & 0.8 \\
\hline 29 & 1.5 & 1.0 & 0.000401 & 0.001517 & 0.5 & 0.040286 & 0.8 \\
\hline 30 & 1.5 & 1.0 & 0.000401 & 0.001517 & 0.5 & 0.053714 & 0.8 \\
\hline 31 & 1.5 & 1.0 & 0.000401 & 0.001517 & 0.5 & 0.067143 & 0.3 \\
\hline 32 & 1.5 & 1.0 & 0.000401 & 0.001517 & 0.5 & 0.067143 & 0.4 \\
\hline 33 & 1.5 & 1.0 & 0.000401 & 0.001517 & 0.5 & 0.067143 & 0.5 \\
\hline 34 & 1.5 & 1.0 & 0.000401 & 0.001517 & 0.5 & 0.067143 & 0.6 \\
\hline 35 & 1.5 & 1.0 & 0.000401 & 0.001517 & 0.5 & 0.067143 & 0.7 \\
\hline
\end{tabular}

It has been reported by Brinnel et al. that parameter $f_{C}$ shows an increased influence on the fracture behaviour for larger values of factor $\kappa$ (i.e. larger than 3) [24]. In order to consider possible interaction effects, additional simulations were performed with values for $\kappa$ between 3.8 and 4.6 . 
Table 5: Additional simulations to include possible interaction effects.

\begin{tabular}{|c|c|c|c|c|c|c|c|}
\hline $\begin{array}{c}\text { Simulation } \\
\text { number }\end{array}$ & $q_{1}$ & $q_{2}$ & $f_{0}$ & $f_{C}$ & $\boldsymbol{f}_{F}$ & $\boldsymbol{f}_{\boldsymbol{N}}$ & $\varepsilon_{N}$ \\
\hline Reference & 1.43 & 0.95 & 0.002 & 0.05 & 0.19 & 0.00018 & 0.3 \\
\hline 36 & 1.444 & 0.95 & 0.002 & 0.05 & 0.19 & 0.00018 & 0.3 \\
\hline 37 & 1.458 & 0.95 & 0.002 & 0.05 & 0.19 & 0.00018 & 0.3 \\
\hline 38 & 1.472 & 0.95 & 0.002 & 0.05 & 0.19 & 0.00018 & 0.3 \\
\hline 39 & 1.486 & 0.95 & 0.002 & 0.05 & 0.19 & 0.00018 & 0.3 \\
\hline 40 & 1.5 & 0.95 & 0.002 & 0.05 & 0.19 & 0.00018 & 0.3 \\
\hline 41 & 1.43 & 0.96 & 0.002 & 0.05 & 0.19 & 0.00018 & 0.3 \\
\hline 42 & 1.43 & 0.97 & 0.002 & 0.05 & 0.19 & 0.00018 & 0.3 \\
\hline 43 & 1.43 & 0.98 & 0.002 & 0.05 & 0.19 & 0.00018 & 0.3 \\
\hline 44 & 1.43 & 0.99 & 0.002 & 0.05 & 0.19 & 0.00018 & 0.3 \\
\hline 45 & 1.43 & 1 & 0.002 & 0.05 & 0.19 & 0.00018 & 0.3 \\
\hline 46 & 1.43 & 0.95 & 0.000125 & 0.05 & 0.19 & 0.00018 & 0.3 \\
\hline 47 & 1.43 & 0.95 & 0.0005 & 0.05 & 0.19 & 0.00018 & 0.3 \\
\hline 48 & 1.43 & 0.95 & 0.000875 & 0.05 & 0.19 & 0.00018 & 0.3 \\
\hline 49 & 1.43 & 0.95 & 0.00125 & 0.05 & 0.19 & 0.00018 & 0.3 \\
\hline 50 & 1.43 & 0.95 & 0.001625 & 0.05 & 0.19 & 0.00018 & 0.3 \\
\hline 51 & 1.43 & 0.95 & 0.002 & 0.00074 & 0.19 & 0.00018 & 0.3 \\
\hline 52 & 1.43 & 0.95 & 0.002 & 0.010592 & 0.19 & 0.00018 & 0.3 \\
\hline 53 & 1.43 & 0.95 & 0.002 & 0.020444 & 0.19 & 0.00018 & 0.3 \\
\hline 54 & 1.43 & 0.95 & 0.002 & 0.030296 & 0.19 & 0.00018 & 0.3 \\
\hline 55 & 1.43 & 0.95 & 0.002 & 0.040148 & 0.19 & 0.00018 & 0.3 \\
\hline 56 & 1.43 & 0.95 & 0.002 & 0.05 & 0.18 & 0.00018 & 0.3 \\
\hline 57 & 1.43 & 0.95 & 0.002 & 0.05 & 0.244 & 0.00018 & 0.3 \\
\hline 58 & 1.43 & 0.95 & 0.002 & 0.05 & 0.308 & 0.00018 & 0.3 \\
\hline 59 & 1.43 & 0.95 & 0.002 & 0.05 & 0.372 & 0.00018 & 0.3 \\
\hline 60 & 1.43 & 0.95 & 0.002 & 0.05 & 0.436 & 0.00018 & 0.3 \\
\hline 61 & 1.43 & 0.95 & 0.002 & 0.05 & 0.5 & 0.00018 & 0.3 \\
\hline 62 & 1.43 & 0.95 & 0.002 & 0.05 & 0.19 & 0 & 0.3 \\
\hline 63 & 1.43 & 0.95 & 0.002 & 0.05 & 0.19 & 0.013429 & 0.3 \\
\hline 64 & 1.43 & 0.95 & 0.002 & 0.05 & 0.19 & 0.026857 & 0.3 \\
\hline 65 & 1.43 & 0.95 & 0.002 & 0.05 & 0.19 & 0.040286 & 0.3 \\
\hline 66 & 1.43 & 0.95 & 0.002 & 0.05 & 0.19 & 0.053714 & 0.3 \\
\hline 67 & 1.43 & 0.95 & 0.002 & 0.05 & 0.19 & 0.067143 & 0.3 \\
\hline 68 & 1.43 & 0.95 & 0.002 & 0.05 & 0.19 & 0.00018 & 0.4 \\
\hline 69 & 1.43 & 0.95 & 0.002 & 0.05 & 0.19 & 0.00018 & 0.5 \\
\hline 70 & 1.43 & 0.95 & 0.002 & 0.05 & 0.19 & 0.00018 & 0.6 \\
\hline
\end{tabular}

Consequently, the total dataset for ANN optimisation consist out of 70 unique input sets with corresponding energy values. Data is shuffled and partitioned in a training fraction $(70 \%)$, a validation fraction (15\%), and testing fraction (15\%).

\section{Results and Discussion}

As can be observed from the summarised results in Figure 7, there is a significant deviation in the predicted fracture energy. The energy values obtained using PS1 and PS4 show the best agreement with experimental data [65]. This interesting observation suggests that the parameter variation in the different parameter sets for the same material, X70 grade steel in this study, can 
have a significant influence on the prediction of fracture toughness. According to the energy results plotted in Figure $7 \mathrm{a}$, the deviations are from 10\% underestimation for PS3 to $32 \%$ overestimation for PS2. Figure 7b shows, as discussed in section 5.2, that the energy variance due to mesh size adjustments between $0.15 \mathrm{~mm}$ and $0.25 \mathrm{~mm}$ is limited.

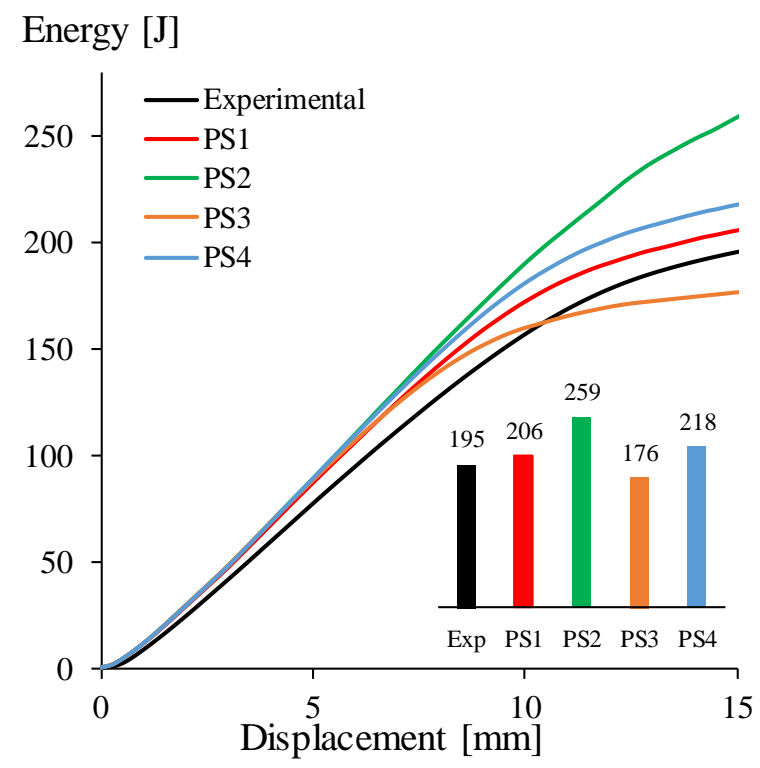

(a)

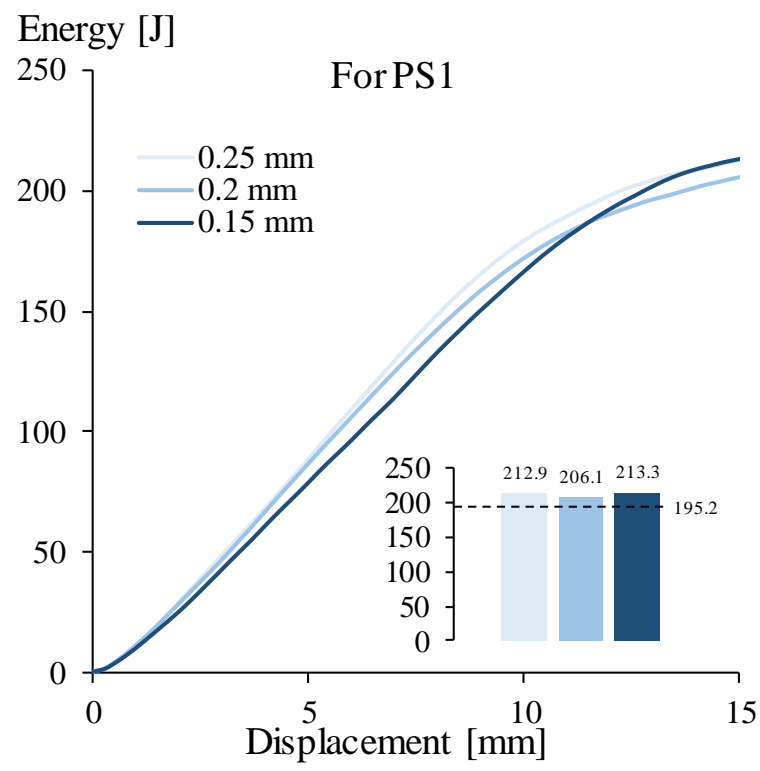

(b)

Figure 7: Fracture energy behaviour of different reference parameter sets for X70 grade steel, as tabulated in Table 2, taken from the literature (a), and energy variations due to mesh size (b) .

Furthermore, the simulated fracture surfaces show noticeable differences for each reference parameter set. As can be seen in Figure 8, the obtained fracture surfaces for PS3 and PS4 show a fracture surface in which a large section of elements is split off from the fracture plane. Since all models are conducted using the exact same mesh, these results suggest that certain damage parameters have a considerable influence on the fracture surface along with the fracture energy.
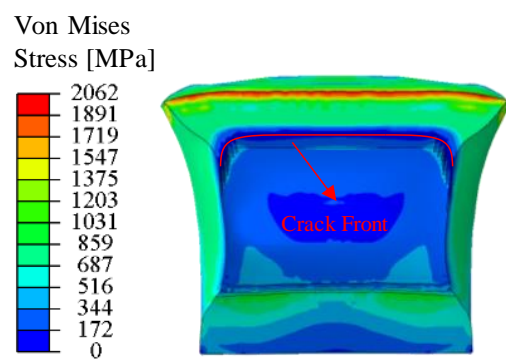

PS1

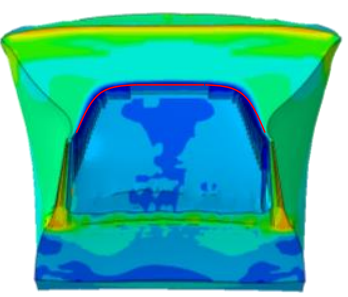

PS2

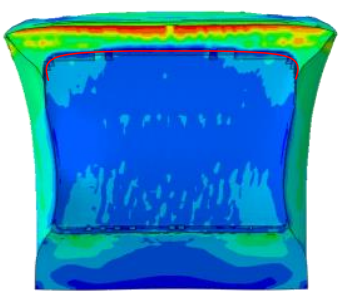

PS3

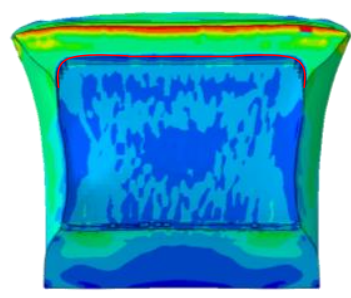

PS4

Figure 8: Obtained fracture surfaces at a striker displacement of $15 \mathrm{~mm}$ for the considered GTN parameter sets. 
As to quantify differences in the observed fracture surfaces, more fracture mechanics parameters such as Crack Tip Opening Displacement (CTOD), Crack Mouth Opening Displacement (CMOD) and Lateral Expansion at the moment of crack initiation are plotted in Figure 9. It is clear that for each model the CTOD, CMOD, and Lateral Expansion indicate different conditions at the fracture initiation. Therefore, it can be stated the variations in damage parameters are significantly influencing the material state at the onset of fracture, thus resulting in different fracture surfaces.

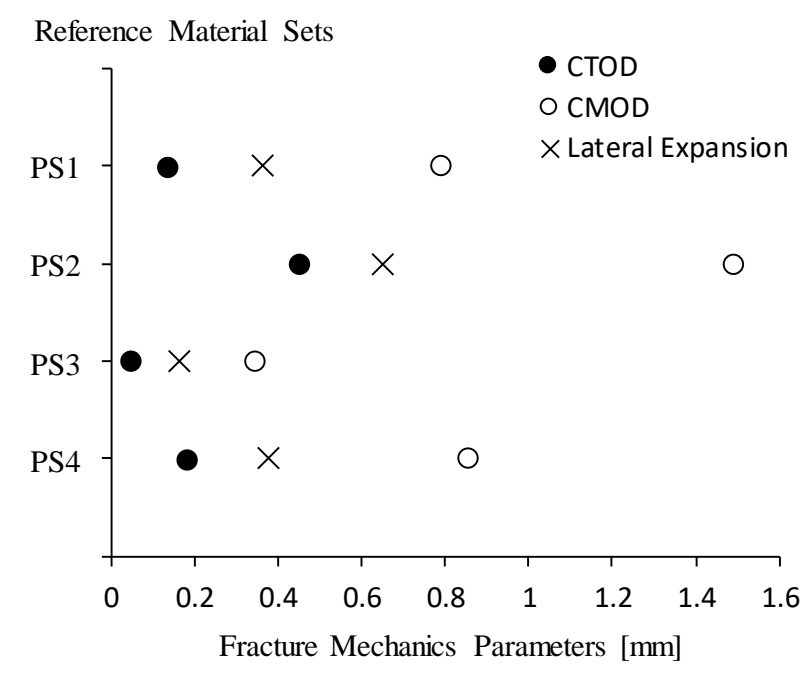

Figure 9: Values for CTOD, CMOD and Lateral Expansion at the point of fracture initiation for each reference parameter set.

Based on the data obtained through the series of numerical simulations, it can be noted that most parameters have a minimal influence on the fracture surface. However, the void volume fraction at failure, $f_{F}$, and the mean value of the nucleation strain, $\varepsilon_{N}$, show a significant influence. In Table 6, an overview is given of the obtained fracture surfaces for the variation in values for $f_{F}$ and $\varepsilon_{N}$. Changes in the observed surfaces can be explained through fracture parameters such as CMOD, CTOD and Lateral Expansion at the moment of crack initiation.

As observed in Table 6, variations in the void volume fraction at failure, $f_{F}$, and the mean value of the nucleation strain, $\varepsilon_{N}$, introduce disparities in the fracture surfaces. Adjusting these variables results in an inconsistent and irregular fracture surface. A small value for parameter $f_{F}$ indicates a small void volume fraction required to initiate element failure. Based on the respective void growth and void nucleation parameters, element failure can be reached prematurely. Low values for $f_{F}$ can result in a deviating crack path, especially due to failure of the critical elements around the notch tip leading to a cascade effect of failing elements. On 
the other hand, reducing $\varepsilon_{N}$ lowers the effective plastic strain at which secondary voids nucleate which accelerates local element failure. Thus, less plastic deformation is required to initiate failure and a local strain peak can result in an aberrant fracture surface with resulting torn-off element groups. It appears that the model sensitivity to local phenomena and aberrations such as singularities occurring during the finite element simulations increases for low values of parameters $f_{F}$ and $\varepsilon_{N}$.

Table 6: Effect of the material-dependent parameters on the resulting fracture surface at a striker displacement of $15 \mathrm{~mm}$ in case of $\kappa<3$.

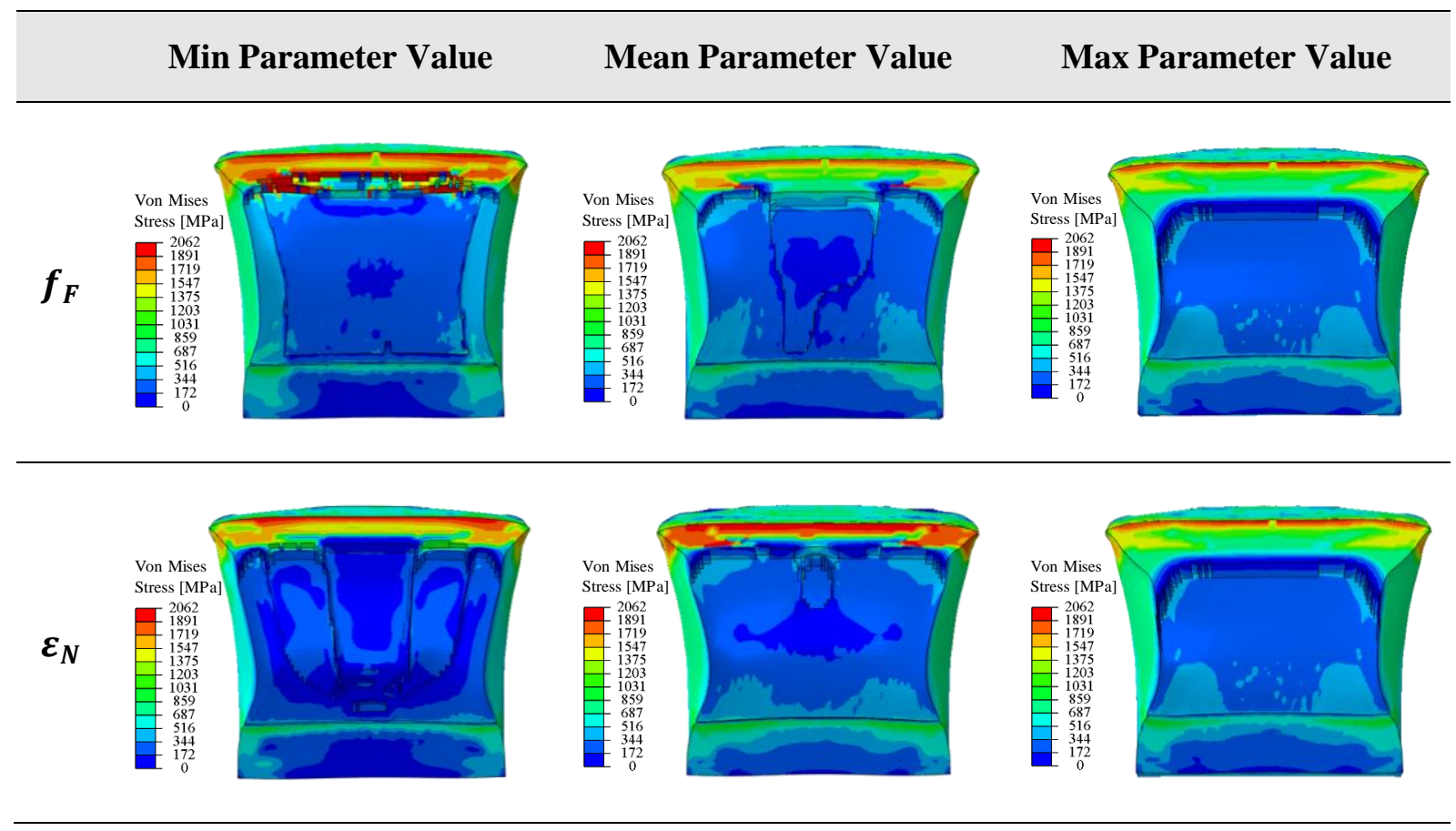

The fracture energy for all numerical simulations are summarised in Figure 10. Once each damage parameter is varied within its value range, the absolute variation of the fracture energy can be analysed. As two reference sets are considered for the cases of $\kappa<3$ and $\kappa>3$ the results are split up with respect to each reference. It is observed that, for the same X70 steel grade the deviation in fracture energy can show large variations.

- $\kappa<3$ :

Resulting fracture energy varies approximately between $-25 \%$ and $+15 \%$ relative to the considered reference - in this case PS1. This indicates that there is a possible spread of $40 \%$ in the predicted fracture energy for the same X70 grade pipeline steel. This large spread of fracture energy predictions can mainly be attributed to parameters $q_{1}$ and $f_{F}$ 
. Even though $f_{c}$ is often considered as crucial for the GTN material model, it presents the smallest spread on the obtained fracture energy.

- $\kappa>3$

Similar to previous situation, variations of fracture energy are large. With a deviation of approximately $-60 \%$ up to $+30 \%$, the spread is even larger. However, the parameters causing the variation are different than in the case of $\kappa<3$.

$\kappa<3$

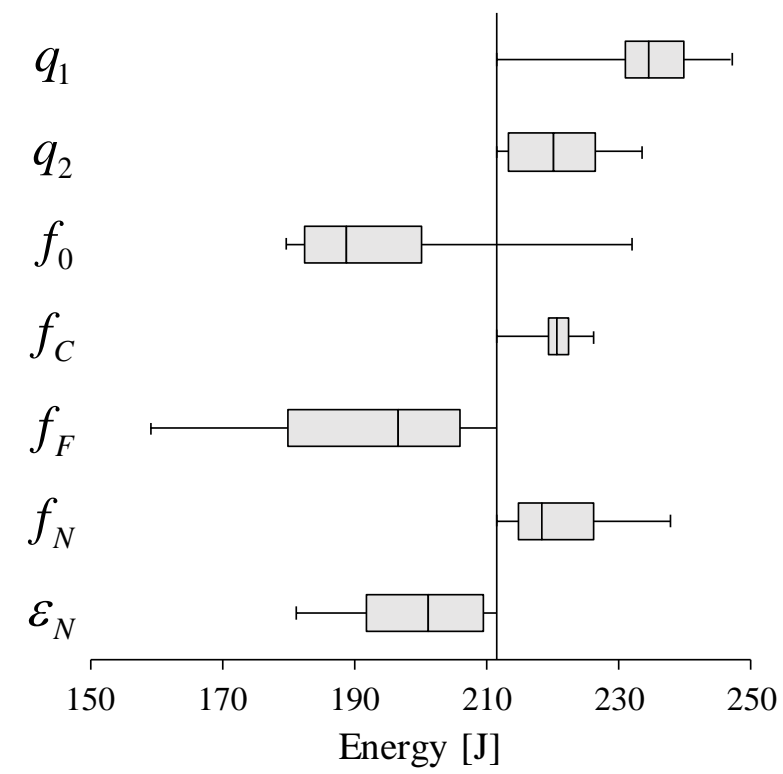

$\kappa>3$

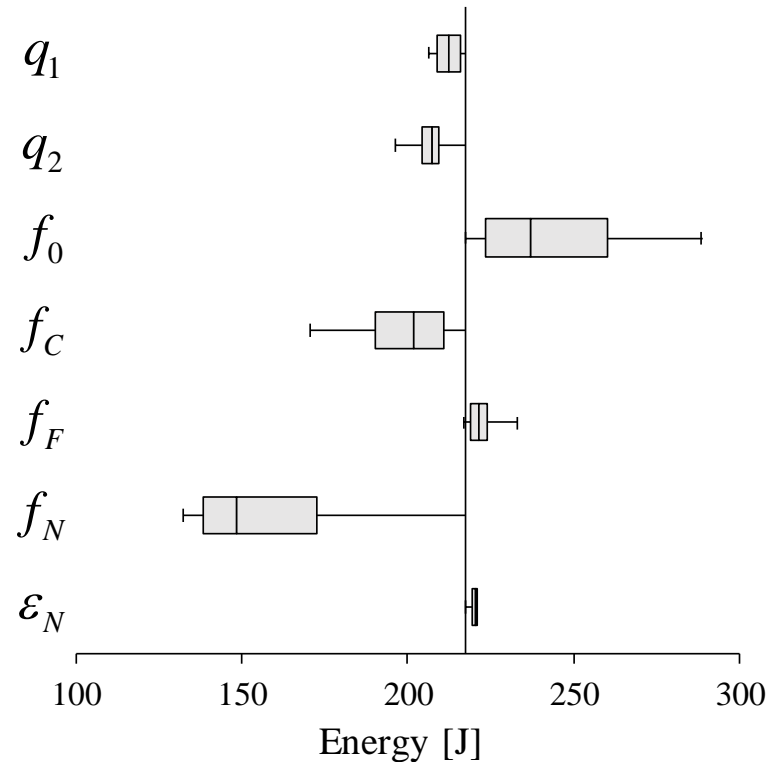

Figure 10: Absolute influence of each GTN parameter over the complete parameter range of X70 grade steel.

However, the relative sensitivity of the fracture toughness with respect to each variable cannot be determined based on this graph since it only considers the absolute energy deviation. Due to the different value ranges, no parameter is changed with the same amount. By considering the size of the actual value ranges, the relative influence for each damage parameter can be determined. In order to obtain the relative influence, an artificial neural network is constructed and numerical data of the simulation series is used to train the system. The dataset used for training of the ANN consists out of the combined simulation data for the cases of $\kappa<3$ and $\kappa>3$.

The weights of the connection between neurons are a measure for the sensitivity of the output variable with respect to each input variable. Thus, by assessing the calibrated weight values of the ANN connections, the relative influence for each damage parameter can be determined. After training and validation, the constructed ANN found a strong relation between the input 
variables and the output energy. By plotting the remaining error for the network as a function of the number of hidden neurons, the optimal number of hidden neurons can be validated. Figure 11 shows that for a network with 8 hidden neurons, the remaining Mean Squared Error (MSE) after training and testing is the lowest.

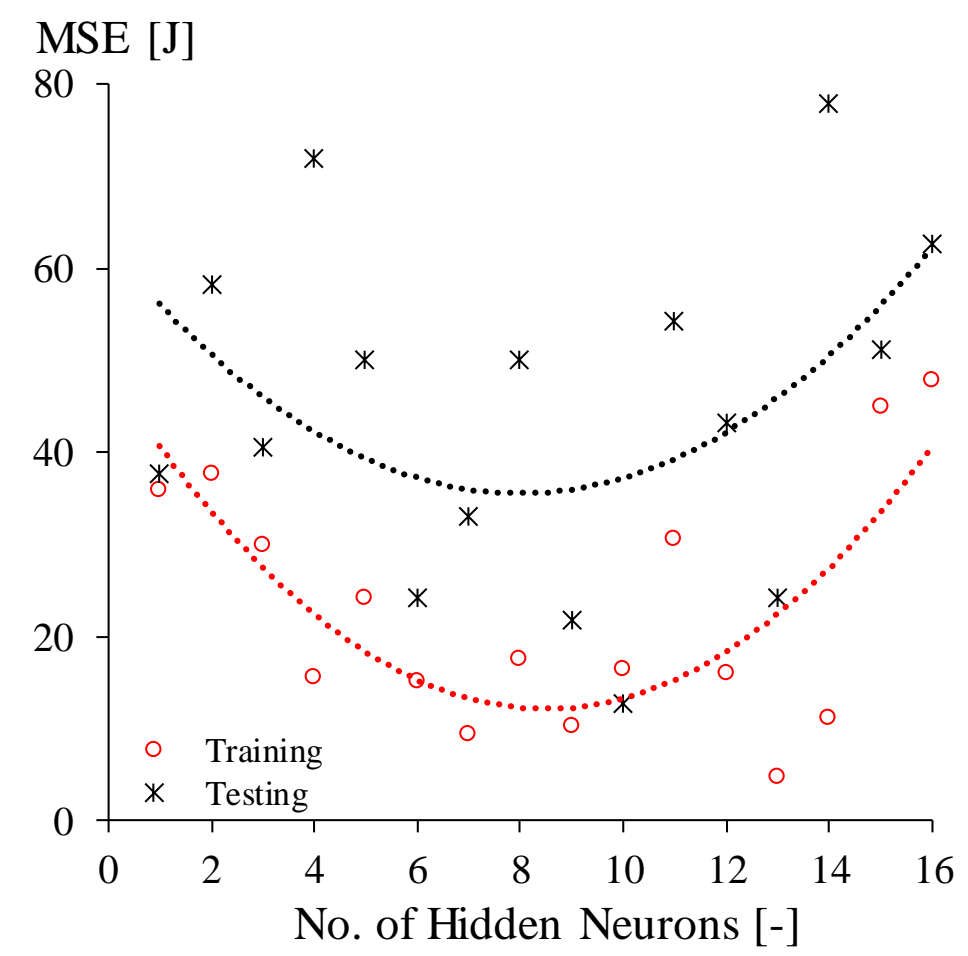

Figure 11: Remaining MSE during training and testing as a function of the number of hidden neurons.

The process of determining the relative influence is repeated three times: for the situation of $\kappa<3, \kappa>3$, and combined $\kappa<3 \& \kappa>3$. As the interaction effects have shown increasing parameter influences for varying $\kappa$ values, it is required to assess the changes in relative importance. Figure 12 shows the performance of the neural networks optimised using the simulation data obtained in the cases of $\kappa<3$ (Figure 12a) and $\kappa>3$ (Figure 12c). The MSE is plotted as a function of each epoch. One epoch represents a period in which the complete data set was fed through the neural network. In these networks each dataset consists out of 35 input sets with corresponding target energy values and are divided into a training fraction (70\%), a validation fraction (15\%), and a testing fraction (15\%). The remaining MSE's for both networks are $12.71 \mathrm{~J}$ and $11.61 \mathrm{~J}$ respectively. This is a deviation of approximately $6 \%$ with respect to the reference values. Also, it can be observed that each network found a strong relation between the target energy value and corresponding energy values obtained throughout the training, 
validation, and testing procedure for both cases $\kappa<3$ (Figure 12b) and $\kappa>3$ (Figure 12d). Subsequently, the relative importance for each parameter is determined.

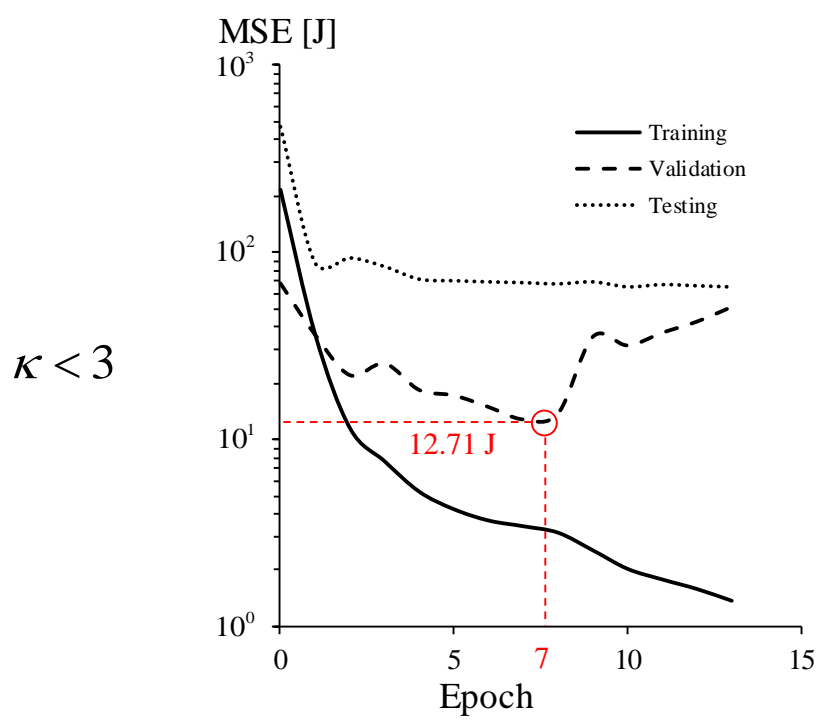

(a)

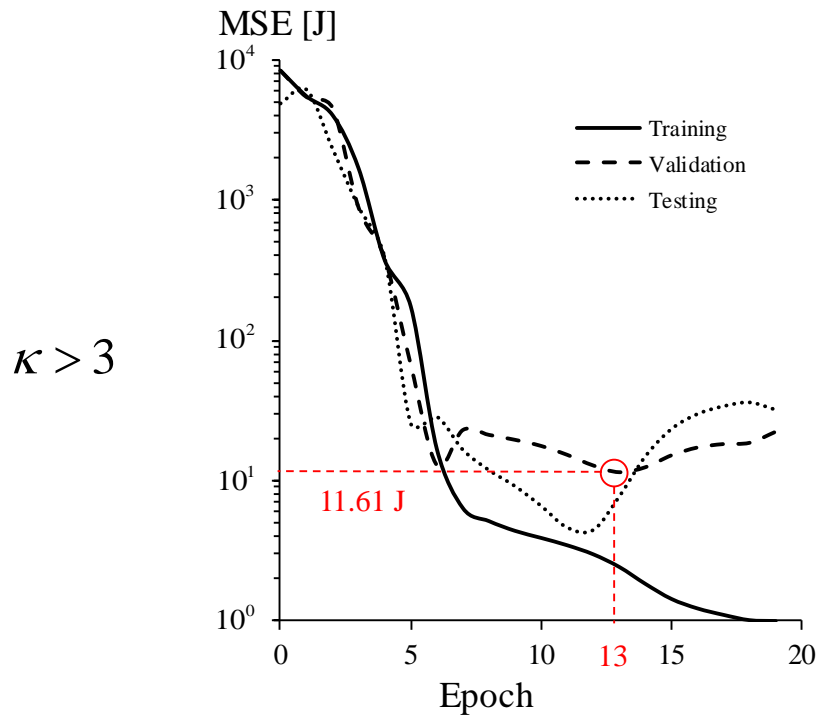

(c)

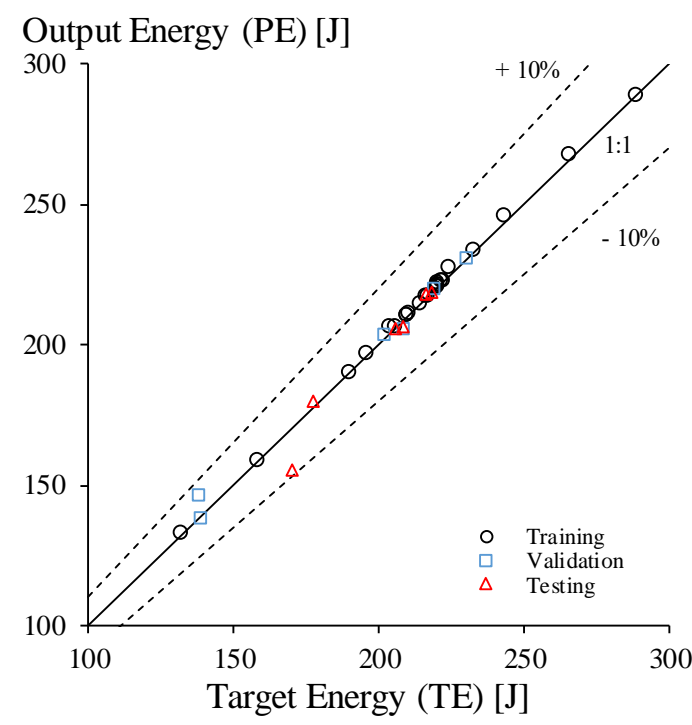

(b)

Output Energy (PE) [J]

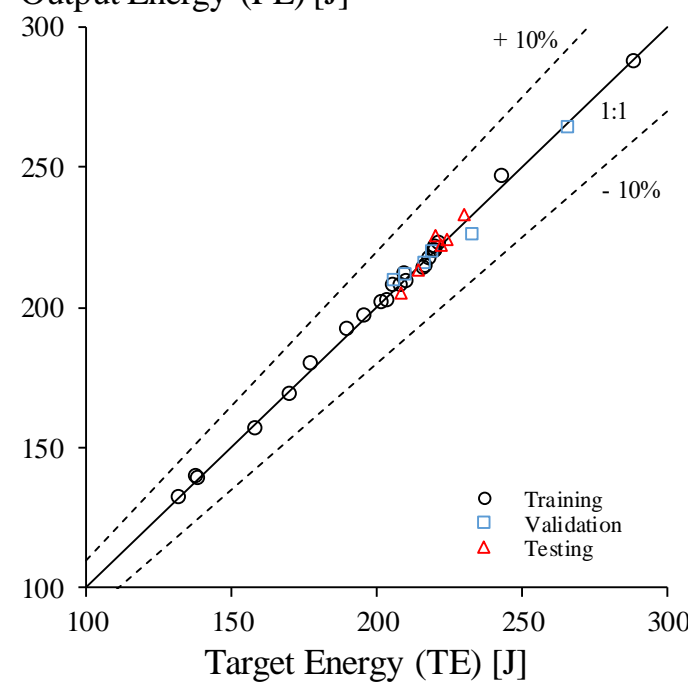

(d)

Figure 12: Performance analysis and regression analysis of the ANN's for $\kappa<3$ (a, b) and $\kappa>3$ (c, d) during training, validation, and testing.

Figure 13 shows that there are critical changes in the relative importance between the two considered cases. It can be observed that in case of $\kappa<3$, parameter $\varepsilon_{n}$ is the dominant parameter whilst, $f_{C}$ - which is often considered as a crucial parameter - has the lowest relative importance. However, for the case of $\kappa>3, f_{C}$ becomes the most influential parameter and the importance of $\varepsilon_{n}$ is greatly reduced. Remarkably, $f_{0}$ and $f_{F}$ are the only parameters that show a high importance in both cases. 

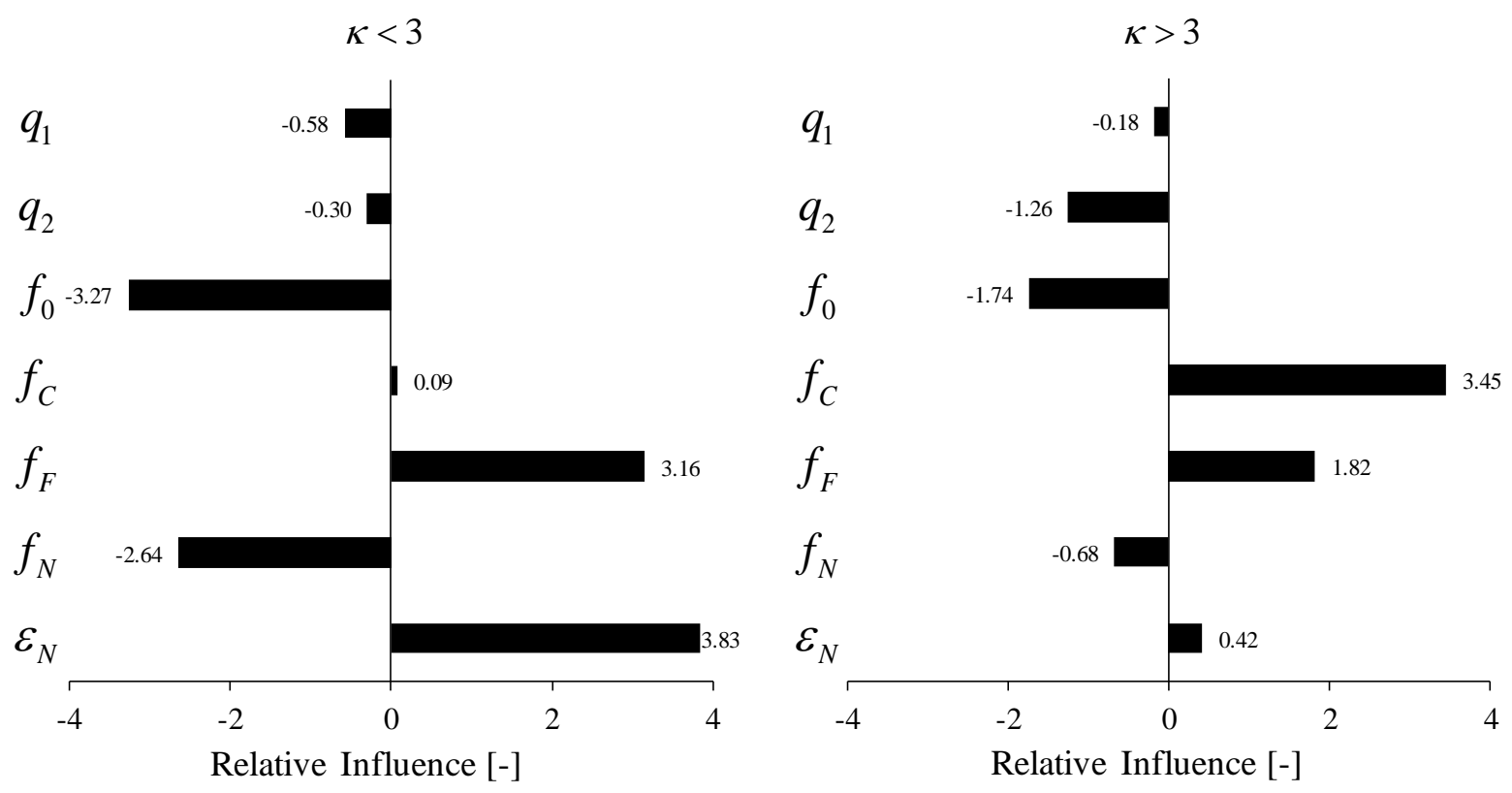

Figure 13: Relative importance for cases of $\kappa<3$ and $\kappa>3$.

It is important to consider the interaction effects between nucleation parameters $f_{N}$ and $\varepsilon_{N}$. Similar to the observations of Brinnel et al. [24] - were parameter $\varepsilon_{N}$ only showed a large influence if parameter $f_{N}$ has a significant value - in this case, $\varepsilon_{N}$ shows a large influence because of the high importance of $f_{N}$. This suggest that, due to the interactions, parameter $f_{N}$ is the controlling factor for the influence of the nucleation parameters.

Ultimately, all data is combined in order to assess the resulting total relative influence for both $\kappa<3 \& \kappa>3$ cases combined. Subsequently, the total dataset consists out of 70 unique input sets with corresponding energy values. Figure 14 visualises the relation found between the predicted fracture energy (PE) by the network and the expected target energy (TE) as extracted from each simulation. 


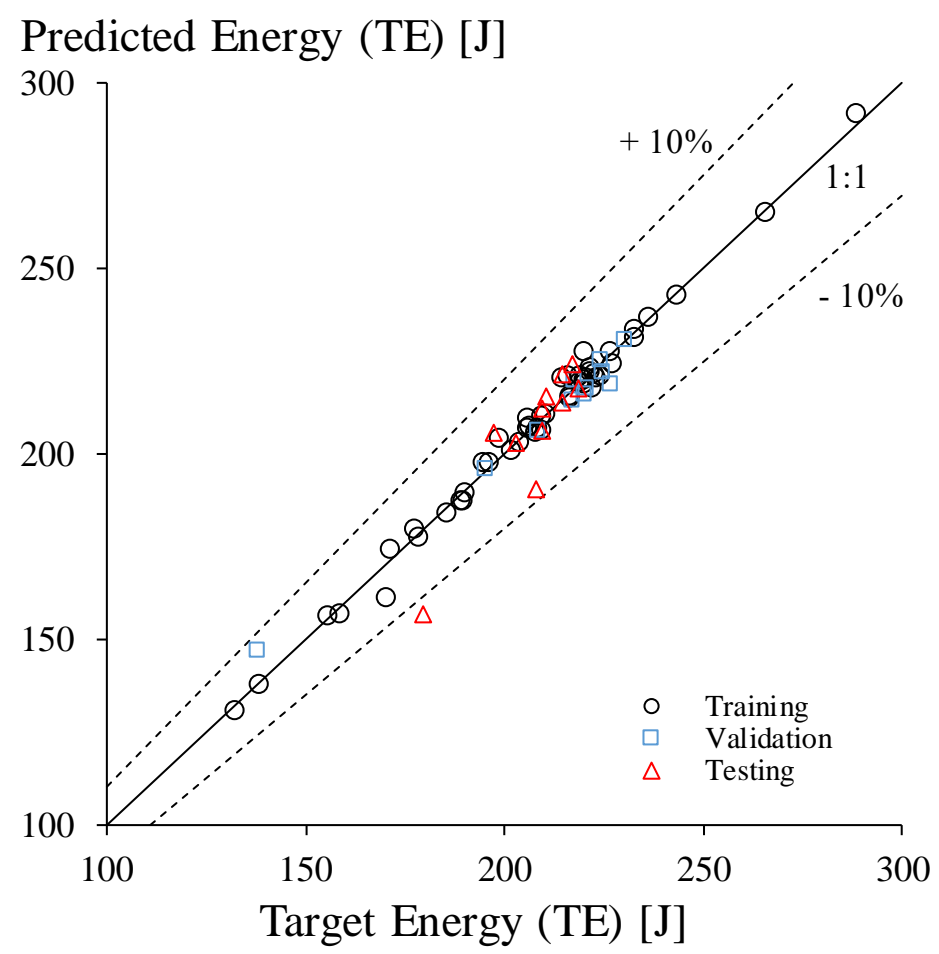

Figure 14: Relation between the predicted energy by the ANN and the target fracture energy during training, validation, and testing.

Figure 15 shows the training process by plotting the residual error for each epoch. Training stopped after 22 epochs, returning an optimal network configuration after 17 epochs with an absolute MSE of 17.48 Joules which is a deviation of approximately $8 \%$ with respect to the considered references. 


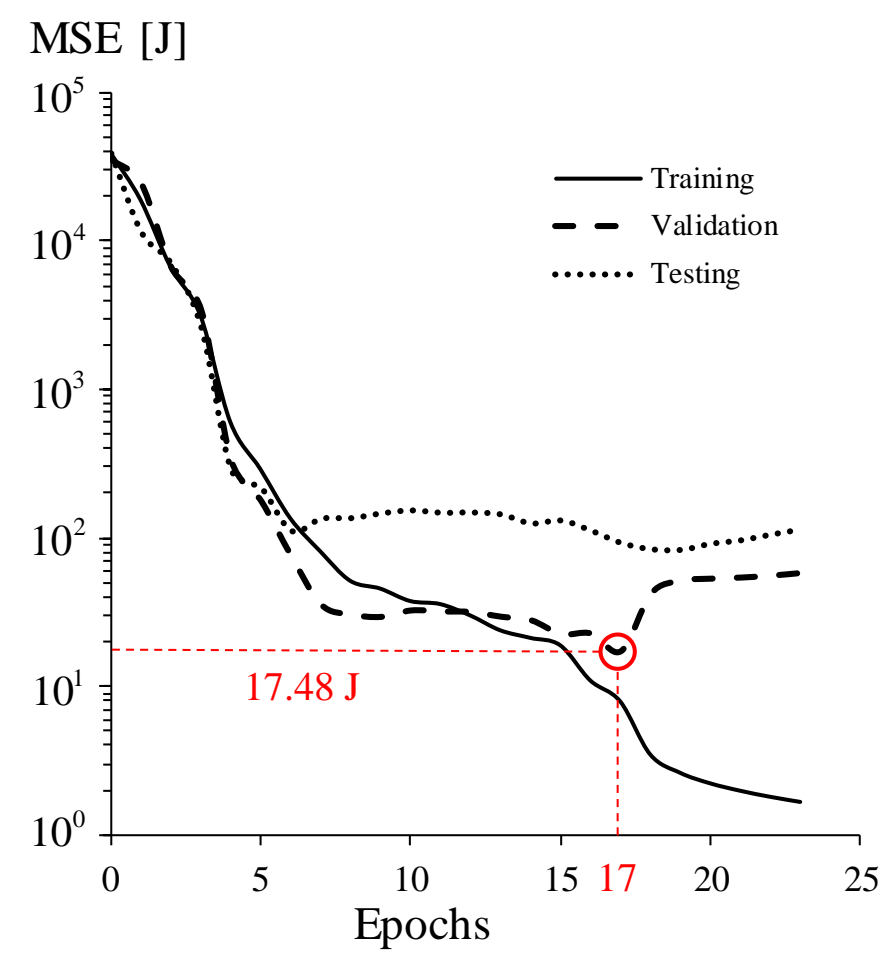

Figure 15: Performance of trained ANN is presented using the remaining MSE after each epoch, a low final MSE indicates a good ANN performance.

Applying the $\mathrm{CW}$ algorithm allows to derive the relative importance from the calibrated connection weights for each input variable. Figure 16 gives an overview of the obtained influence for each material parameter with respect to the fracture energy. It is observed that $f_{0}$ and $f_{C}$ show the highest relative contributions. Whilst $q_{2}$ and $\varepsilon_{N}$ show the lowest contributions. In other words, adjusting parameters $f_{0}$ and $f_{C}$ with the same amount will lead to a large but similar deviation in fracture energy. This data allows to compare the influence of each damage parameter with each other. Adjusting parameters $q_{1}$ and $f_{F}$ with the same amount will yield approximately the same results in terms of the obtained fracture energy. On the other hand, adjusting the value of $q_{1}$ will have approximately four times more effect on the final energy value. Furthermore - based on this data - parameter $f_{0}$ can be considered as important as critical parameter $f_{C}$. 


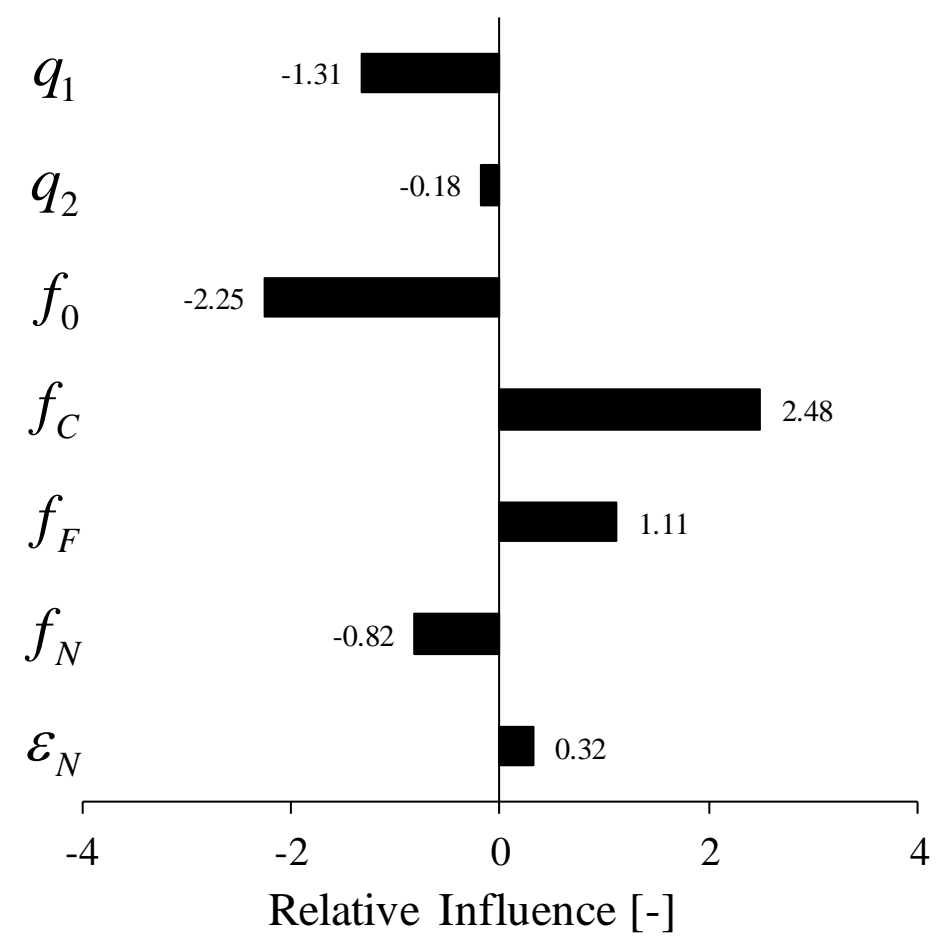

Figure 16: Relative influence of the material-dependent GTN parameters on the fracture energy.

Summarised, the data presented in Figure 16, suggests there are two parameters $\left(f_{0}\right.$ and $\left.f_{C}\right)$ with a dominant influence, three parameters $\left(q_{1}, f_{F}\right.$, and $\left.f_{N}\right)$ with a medium influence, and two parameters $\left(q_{2}\right.$ and $\left.\varepsilon_{N}\right)$ with a low influence on the fracture energy. However, in this study $f_{F}$ and $\varepsilon_{N}$ have shown influence on both the fracture surface appearance as well as on the energy prediction thus requiring additional consideration. Table 7 summarises the obtained relative influences for all considered cases. Based on the observed parameter contributions it is suggested that parameters $f_{0}, f_{C}, f_{F}$, and $f_{N}$ require careful consideration. Practically, this implicates that these parameters should be determined for each individual application of the GTN damage model..

Table 7: Overview of obtained relative importance for each parameter with corresponding rank, 1 being the highest importance and 7 being the lowest importance.

\begin{tabular}{c|ccccccc} 
& $\boldsymbol{q}_{\mathbf{1}}$ & $\boldsymbol{q}_{\mathbf{2}}$ & $\boldsymbol{f}_{\mathbf{0}}$ & $\boldsymbol{f}_{\boldsymbol{C}}$ & $\boldsymbol{f}_{\boldsymbol{F}}$ & $\boldsymbol{f}_{\boldsymbol{N}}$ & $\boldsymbol{\varepsilon}_{\boldsymbol{N}}$ \\
\hline$\kappa<3$ & -0.58 & -0.30 & -3.27 & 0.09 & 3.16 & -2.64 & 3.83 \\
Rank & 6 & 5 & 2 & 7 & 3 & 4 & 1 \\
$\kappa>3$ & -0.18 & -1.26 & -1.74 & 3.45 & 1.82 & -0.68 & 0.42 \\
Rank & 7 & 4 & 3 & 1 & 2 & 5 & 6 \\
\hline Combined & -1.31 & -0.18 & -2.25 & 2.48 & 1.11 & -0.82 & 0.32 \\
Rank & 3 & 7 & 2 & 1 & 4 & 5 & 6
\end{tabular}


Also, note that for one of the most influential material parameters observed in this investigation, $f_{0}$, there is no consistent way of determining its correct value. As mentioned in previous sections, this material parameter can be calibrated, calculated or obtained from the literature.

It is fair to state that the different investigations reporting on the GTN material parameters for X70 grade steel, result in a deviating fracture toughness prediction. One of the possible explanations for the difference in damage parameter sets is the use of different calibration procedures and optimisation processes. This indicates the importance of selecting the calibration and optimisation procedure according to the relevant stress states in the application of interest.

Besides extracting the relative importance for each input parameter, the trained ANN allows to make predications using a given input parameter set. As a means of validation, the reference sets PS1, PS2, PS3, and PS4 are fed to the network which resulted in a predicted fracture energy. Figure 17 shows the ANN energy prediction and target energy value obtained from simulations. It is observed that the ANN is able to approximate the energy values within $10 \%$ of the target value.

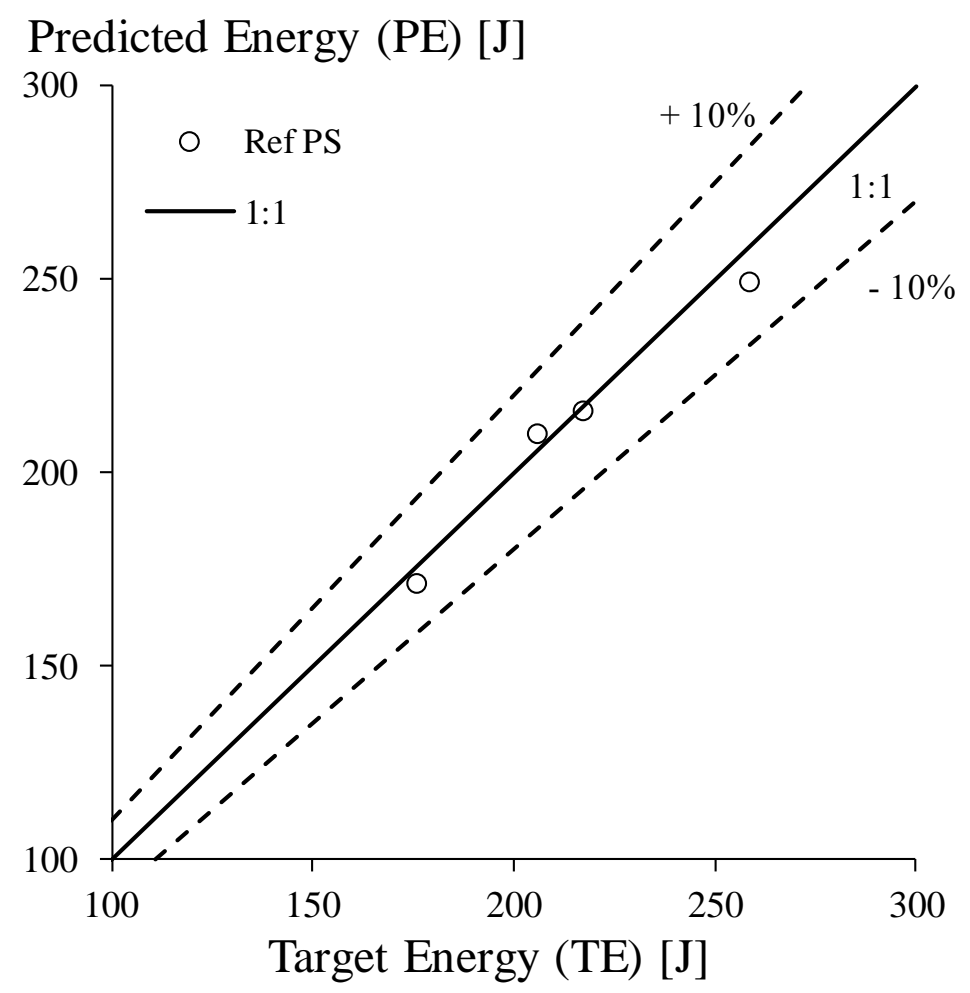

Figure 17: Predictions of ANN for each considered reference parameter set for the GTN damage model. 
This data suggest that it is possible to have obtain satisfactory approximations of the CVN energy using the trained ANN for a random input set. It is important to note that this statement is only valid if the random input set only considers input values within the respective parameter value ranges. Using this machine learning approach, an initial guess for the fracture energy can be made without running a new finite element model. Of course, the constrains and mechanical background of the GTN damage still has to be respected when implementing the random input parameter set. Inverting this trained ANN using optimisation algorithms would allow to generate an GTN input set for a desired energy value. However, this procedure falls outside the scope of this investigation. 


\section{Conclusions}

In this investigation, the influences of the GTN damage parameters were quantified in the case of X70 grade pipeline steel. Relative influences of the constitutive parameters $q_{1}$ and $q_{2}$, initial void volume fraction, $f_{0}$, critical void volume fraction, $f_{c}$, void volume fraction at failure, $f_{F}$ , void volume fraction of nucleated voids, $f_{N}$, and the mean value of the nucleation strain, $\varepsilon_{N}$ , were determined through a machine learning based approach. An ANN was trained and optimised using the data obtained from finite element simulations. Using the $\mathrm{CW}$ algorithm, relative importance for each considered material parameter was be derived. The use of an artificial neural network in this work has shown to be a performant tool in assessing a complex non-linear problem. With a relatively limited data set, it was possible to calibrate and train an artificial neural network with an acceptable residual mean square error. Furthermore, the ANN can be applied to generate acceptable energy predictions for new GTN parameter sets.

It was observed that in this case study, parameter $f_{C}$ is the most influential parameter regarding the fracture energy. In most cases, this variable is considered as a critical parameter that should be calibrated for each individual case. However, when considering the case of $\kappa<3, f_{C}$ yields the lowest influence relative to the other damage parameters. In this case, parameter $\varepsilon_{N}$ shows the highest importance. Since the distribution of the nucleation strain cannot be determined practically, it is often assumed to have a typical value. In some cases, it is adjusted to fit numerical data with experimental results. Consequently, the numerical fracture energy can be easily adjusted to fit the experimental with relatively limited changes to parameter $\varepsilon_{N}$. However, the importance $\varepsilon_{N}$ cannot be evaluated individually due to the interaction effects between the nucleation parameters. Based on the data, it is suggested that the importance of $f_{N}$ is the controlling factor for the importance of the nucleation parameters. It should also be noted that $f_{0}$ is the sole parameter that maintains its importance throughout the range of $\kappa$.

The predictive performance GTN damage model is highly dependent on material parameters that can be obtained from the literature and adjusted if necessary to fit the required behaviour. Moreover, if the parameter is to be calibrated, a calibration procedure should be selected based on the stress conditions present in the desired application. Also, the acceleration of the void volume growth with factor $\kappa$ should be considered. The obtained data shows that the relative importance for each parameter is highly dependent on $\kappa$. 
The GTN damage model is widely accepted and used as a damage model to simulate ductile fracture propagation. It is implemented in a variety of software packages and model parameters are available in literature for an extensive set of materials. However, this investigation shows that the material-dependent parameters in the literature should be used with the necessary caution:

The material-dependent parameters should be determined in a consistent way. In this study, critical parameter $f_{C}$ showed the least relative influence on the fracture energy in case of $\kappa<3$ . As $f_{C}$, in some cases, is the only calibrated damage parameter, numerical fracture behaviour can easily be fitted with experimental data by applying small adjustments to the most influential parameters $\left(f_{0}, f_{F}, f_{N}\right.$, and $\left.\varepsilon_{N}\right)$. In general, based on the results obtained in this study, it is suggested to validate or calibrate parameters $f_{0}, f_{C}, f_{F}$, and $f_{N}$ for each individual application as these variables have shown significant influence with respect to the fracture energy.

Future research using AI in fracture mechanics investigations yields a lot of potential towards fracture predictions. The procedure applied in this study allowed to determine the relative importance of each parameter and showed promising results regarding its predictive performance. It is shown that a shallow neural network is able to produce acceptable energy predictions for a given GTN input set. This type of network can be inverted in order to reverse the process which would result in a new GTN input set for a required energy value. In the future, AI procedures could be applied to facilitate the calibration procedure of existing damage models.

\section{Acknowledgements}

The author gratefully acknowledges the support of the Research Foundation Flanders (FWO) via $\mathrm{PhD}$ fellowship grant $1 \mathrm{SB} 6420 \mathrm{~N}$. 


\section{References}

[1] A. R. Duffy, "Full scale studies," in A.G.A. Symposium on Line Pipe Research, Cat. No. L30 075, 1966.

[2] R. J. Eiber, "Fracture propagation," in A.G.A 4th Symposium on Line Pipe Research, Cat. No. L30 075, 1969.

[3] G. D. Fearnehough, "Fracture Propagation Control in Gas Pipelines: A Survey of Relevant Studies," International Journal of Pressure Vessels and Piping, vol. 2, no. 4, pp. 257-282, 1974.

[4] J. Besson, "Continuum models of ductile fracture: A review," International Journal of Damage Mechanics, no. 19, pp. 3-52, 2010.

[5] ASTM International, "ASTM E23-18: Standard Test Methods for Notched Bar Impact Testing of Metallic Materials,” ASTM International, West Conshohocken, 2018.

[6] X. K. Zhu and B. N. Leis, "CVN and DWTT Energy methods for determining fracture arrest toughness of high strength pipeline steels," in Proceedings of the 9th International Pipeline Conference, Calgary, Canada, 2012.

[7] A. Cosham, R. M. Andrews and T. Schmidt, "The EPRG Recommendation for Crack Arrest Toughness for Line Pipe Steel,” European Pipeline Research Group, Germany, 2019.

[8] W. A. Maxey, "Fracture Initiation, Propagation and Arrest," in Proceedings of 5th Symposium on Line Pipe Research, Houston (USA), 1974.

[9] G. Mannucci, G. Demofonti and M. Di Biagio, "X100 - Fracture initiation and propagation,” ECSC Report, 2005.

[10] G. Demofonti, G. Mannucci, L. F. Di Vito, R. Aristotile, M. Di Biaggio, G. Malatesta, D. Harris and P. L. Harrison, "Ultra-high-strength pipeline prototyping for natural gas transmission DEMOPIPE," Research Programme of the Research Fund for Coal and Steel RDT, 2005. 
[11] X.-K. Zhu, "State-of-the-art review of fracture control technology for modern and vintage gas transmission pipelines," Engineering Fracture Mechanics, no. 148, pp. 260$280,2015$.

[12] ISO, "ISO 3183:2019 - Petroleum and Natural gas industries-Steel pipe for pipeline transportation systems," International Organization for Standardization, 2019.

[13] J. Lian, M. Sharaf, F. Archie and S. Münstermann, “A hybrid approach for modelling of plasticity and failure behaviour of advanced high-strength steel sheets," International Journal of Damage Mechanics, vol. 22, no. 2, pp. 188-218, 2013.

[14] A. Kami, B. Mollaei Dariani, D. Sorin Comsa, D. Banabic, A. Sadough Vanini and M. Liewald, "Calibration of GTN Damage Model Parameters Using Hydraulic Bulge Test," The Romanian Journal of Technical Sciences Applied Mechanics, no. 61, pp. 245-260, 2016.

[15] R. Kiran and K. Khandelwal, "Gurson model parameters for ductile fracture simulation in ASTM A992 steels," Fatigue \& Fracture of Engineering Materials \& Structures, vol. 2, no. 37, pp. 1-13, 2013.

[16] P. Thibaux and F. Van den Abeele, "Determination of crack initiation and propagation energy in instrumented Charpy V-notch impact tests by finite element simulations," in Pipeline Technoogy Conference, Ostend, 2009.

[17] F. Rivalin, J. Besson, A. Pineau and M. Di Fant, "Ductile tearing of pipeline-steel wide plates II. Modeling of in-plane crack propagation," Engineering Fracture Mechanics, no. 68 , pp. 347-364, 2001.

[18] D. Z. Sun, D. Siegele, B. Voss and W. Schmitt, “Application of local damage models to the numerical analysis of ductile rupture," Fatigue \& Fracture of Engineering Materials and Structures, no. 2, pp. 210-212, 1989.

[19] I.-J. Kim, Y.-C. Jang, C.-M. Kim, Y.-P. Kim, Y.-Y. Jang and N.-S. Huh, “Crack growth simulation of fracture toughness specimens using GTN (Gurson-Tvergaard-Needleman) model," in Proceedings of KSME Spring Conference, Jeongseon-gun, 2018. 
[20] Y. Chen and S. Lambert, "Analysis of ductile tearing of pipeline-steel in single edge notch tension specimen," International Journal of Fracture, no. 124, pp. 179-199, 2003.

[21] Y. Chen and S. Lambert, "Numerical modeling of ductile tearing for semi-elliptical surface cracks in wide plates," International Journal of Pressure Vessels and Piping, no. 82 , pp. 417-426, 2005.

[22] L. Ying, W. Liu, D. Wang and P. Hu, "Parameter Calibration of GTN Damage Model and Formability Analysis of 22MnB5 in Hot Forming Process," Journal of Materials Engineering and Performance, vol. 26, no. 11, pp. 5155-5165, 2017.

[23] J. Faleskog, X. Gao and C. F. Shih, "Cell model for nonlinear fracture analysis - I. Micromechanics calibration," International Journal of Fracture, no. 89, pp. 355-373, 1998.

[24] V. Brinnel, J. Langenberg, F. Kordtomeikel, Y. Di and S. Münstermann, "Numerical derivation of strain-based criteria for ductile failure: Discussions on sensitivity and validity,” Engineering Fracture Mechanics, vol. 148, pp. 421-440, 2015.

[25] S. Nasiri, M. Reza Khosravani and K. Weinberg, "Fracture mechanics and mechanical fault detection by artificial intelligence methods: A review," Engineering Failure Analysis, no. 81, pp. 270-293, 2017.

[26] M. Abendroth and M. Kuna, "Identification of ductile damage and fracture parameters from the small punch test using neural networks," Engineering Fracture mechanics, vol. 73, pp. 710-725, 2006.

[27] A. Needleman and V. Tvergaard, "An Analysis of Ductile Rupture Modes at a Crack Tip," Journal of the Mechanics and Physics of Solids, vol. 2, no. 35, pp. 151-183, 1987.

[28] J. Gurland and J. Plateau, "The mechanism of ductile rupture of metals containing inclusions," Trans. ASM, no. 56, pp. 442-454, 1963.

[29] A. L. Gurson, "Continuum Theory of Ductile Rupture by Void Nucleation and Growth: Part I - Yield Criteria and Flow Rules for Porous Ductile Media," Journal of Engineering Materials and Technology, vol. 1, no. 99, pp. 2-15, 1977. 
[30] V. Tvergaard, "Influence of Voids on Shear Band Instabilities Under Plane Strain Conditions," International Journal of Fracture, no. 17, pp. 389-407, 1981.

[31] V. Tvergaard, "On Localization in Ductile Materials Containing Spherical Voids," International Journal of Fracture, no. 18, pp. 237-252, 1982.

[32] V. Tvergaard and A. Needleman, "Analysis of the cup-cone fracture in a round tensile bar," Acta Metallurgica, vol. 1, no. 32, pp. 157-169, 1984.

[33] G. Hütter and L. Zybell, "Models Describing Void Growth," in Recent Trends in Fracture and Damage Mechanics, Springer, 2016, pp. 376-385.

[34] C. C. Chu and A. Needleman, "Void Nucleation Effects in Biaxially Streched Sheets," Journal of Engineering Materials and Technology, vol. 3, no. 102, pp. 249-256, 1980.

[35] A. Needleman and V. Tvergaard, "An analysis of dynamic ductile crack growth in a double edge cracked specimen," International Journal of Fracture, no. 49, pp. 41-67, 1991.

[36] V. Tvergaard, "Influence of void nucleation on ductile shear fracture at a free surface," Journal of the Mechanics and Physics of Solids, vol. 6, no. 30, pp. 399-425, 1982.

[37] W. Brocks, D. Z. Sun and A. Honig, "Verification of the transferability of micromechanical parameters by cell model calculation with visco-plastic materials," International Journal of Plasticity, no. 11, pp. 971-989, 1995.

[38] J. Kim, X. Gao and S. Srivatsan, "Modeling of void growth in ductile solids: effects of stress triaxiality and initial porosity," Engineering Fracture Mechanics, no. 71, pp. 379400, 2004.

[39] A. G. Franklin, "Comparison between quantitative microscope and chemical methods for assessment of non-metallic inclusions," Journal of the Iron and Steel institute, no. 207, pp. 181-186, 1969.

[40] L. Xia, C. Fong Shih and J. W Hutchinson, "A computational approach to ductile crack growth under large scale yielding conditions," Journal of Mechanics and Physics of Solids, vol. 3, no. 43, pp. 389-413, 1995. 
[41] Z. L. Zhang, C. Thaulow and J. Odegard, "A complete Gurson Model Approach for Ductile Fracture," Engineering Fracture Mechanics, vol. 2, no. 67, pp. 155-168, 2000.

[42] W. Wcislik, "Experimental determination of critical void volume fraction $\mathrm{fF}$ for the Gurson Tvergaard Needleman (GTN) model," in 21st European Conference on Fracture, Catania, Italy, 2016.

[43] W. W. Zhang and S. Cong, "Failure analysis of SUS304 sheet during hydro-bulging based on GTN ductile damage model," The International Journal of Advanced Manufacturing Technology, vol. 86, pp. 427-435, 2016.

[44] F. Rahimidehgolan, G. Majzoobi, F. Alinejad and J. Fathi Sola, "Determination of the Constants of GTN Damage Model Using Experiment, Polynomial Regression and Kriging Methods,” Applied Sciences, no. 11, pp. 1179-1199, 2017.

[45] P. Zhang, M. Pereira, B. Rolfe, D. Wilkosz, B. Abeyrathna and M. Weiss, "A new strain-based method to determine GTN parameters for thin stainless steel foil," in International Conference and Workshop on Numerical Simulation of 3D Sheet Metal Forming Processes, Tokyo, 2018.

[46] D. Banabic, "Determination of GTN Parameters from Uniaxial Tests," in Multiscale Modelling in Sheet Metal Forming, Springer, 2016, pp. 142-143.

[47] R. Kiran and K. Khandelwal, "Gurson model parameters for ductile fracture simulation in ASTM A992 steels," Fatigue \& Fracture of Engineering Materials \& Structures, no. 37, pp. 171-183, 2014.

[48] H. Li and M. Fu, "Calibration of the Ductile Fracture Models," in Deformation-Based Processing of Materials: Behavior, Performance, Modeling, and Control, Elsevier, 2019, pp. 121-131.

[49] L. Yeda, W. Renqiang, Z. Bing and F. Tao, “Application of GTN Model in Tensile Fracture of Pipeline Steel,” Key Engineering Materials, no. 777, pp. 451-456, 2018.

[50] F. Abbassi, T. Belhadj, S. Mistou and A. Zghal, "Parameter identification of a mechanical ductile damage using Artificial Neural Networks in sheet metal forming," Materials \& Design, vol. 45, pp. 605-615, 2012. 
[51] A. M. Turing, "Computing Machinery and Intelligence," Mind, no. 49, pp. 433-460, 1950.

[52] S. Russel and P. Norvig, Artificial Intelligence: A Modern Approach, New Jersey: Pearson, 1995.

[53] F. Rosenblatt, "The Perceptron: A Probabilistic Model for Information Storage and Organisation in the Brain," Pschycological Review, vol. 6, no. 65, pp. 386-408, 1958.

[54] D. Von Segger, CRC Standard Curves and Surfaces, Boca Raton: CRC Press, 1993.

[55] MathWorks, MATLAB release 2019a, Massachusetts, United States.

[56] R. Ramadevi, R. B. Sheela and V. Prakash, "Role of Hidden Neurons in an Elman Recurrent Neural Network in Classification of Cavitation Signals," International Journal of Computer Applications, vol. 37, no. 7, pp. 9-13, 2012.

[57] K. Gnana Sheela and S. N. Deepa, "Review on Methods to Fix Number of Hidden Neurons in Neural Networks," Mathematical Problems in Engineering, vol. 2013, no. 6, 2013.

[58] J. D. Olden and D. A. Jackson, "Illuminating the "black box": a randomization approach for understanding variable contributions in artificial neural networks," Ecological Modelling, no. 154, pp. 135-150, 2002.

[59] J. D. Olden, M. K. Joy and R. G. Death, "An accurate comparison of methods for quantifying variable importance in artificial neural networks using simulated data," Ecological Modelling, no. 178, pp. 389-397, 2004.

[60] K. Levenberg, "A Method for the Solution of Certain Non-Linear Problems in Least Squares," Quarterly of Applied Mathematics, vol. 2, no. 2, pp. 164-168, 1944.

[61] D. W. Marquardt, “An Algorithm for the Least-Squares Estimation of Nonlinear Parameters," SLAM Journal of Applied Mathematics, vol. 11, no. 2, pp. 431-441, 1963.

[62] Dassault Systèmes, ABAQUS Documentation 2019, Providence, United States. 
[63] R. Talemi, S. Cooreman and D. Van Hoecke, "Finite element simulation of dynamic brittle fracture in pipeline steel: A XFEM-based cohesive zone approach," Journal of Materials, Design and Applications, vol. 5, no. 232, pp. 357-370, 2018.

[64] G. Cricri, “A Consistent use of the Gurson-Tvergaard-Needleman damage model for the R-curve calculation,” Frattura ed Integrita Strutturale, vol. 24, pp. 161-174, 2013.

[65] H. Sayyed, “Apportion of Charpy energy in API 5L grade X70 pipeline steel," International Journal of Pressure Vessels and Piping, no. 85, pp. 879-884, 2008. 